\title{
1 Protective efficacy of an orf virus-vector encoding the hemmagglutinin and the nucleoprotein of
}

2 influenza $A$ virus in swine

3

4 Lok R. Joshi ${ }^{1,2}$, David Knudsen ${ }^{2}$, Pablo Pineyro ${ }^{3}$, Santhosh Dhakal ${ }^{4}$, Gourapura J. Renukaradhya ${ }^{4}$, Diego G.

5 Diel ${ }^{1,2 \#}$

6

$7 \quad{ }^{1}$ Department of Population Medicine and Diagnostic Sciences, Animal Health Diagnostic Center, College

8 of Veterinary Medicine, Cornell University, Ithaca, New York, USA.

9

$10{ }^{2}$ Department of Veterinary and Biomedical Sciences, Animal Disease Research And Diagnostic

11 Laboratory, South Dakota State University, Brookings, SD, United States.

12

$13{ }^{3}$ Department of Veterinary Diagnostic and Production Animal Medicine, lowa State University, Ames, 14 lowa, USA.

15

$16{ }^{4}$ Department of Veterinary Preventive Medicine, Center for Food Animal Health, Ohio State University, 17 Wooster, Ohio, USA.

18

19

\#Corresponding author

20

E-mail: dgdiel@cornell.edu

21

22

23

24 


\section{Abstract}

27 Swine influenza is a highly contagious respiratory disease of pigs caused by influenza A viruses (IAV-S). IAV-S causes significant economic losses to the swine industry and poses constant challenges to public health due to its zoonotic potential. Thus effective IAV-S vaccines are highly desirable and would benefit both animal and human health. Here, we developed two recombinant orf viruses, expressing the hemagglutinin (HA) gene (OV-HA) or both the HA and the nucleoprotein (NP) genes of IAV-S (OV-HA-NP). The immunogenicity and protective efficacy of these two recombinant viruses were evaluated in pigs. Both OV-HA and OV-HA-NP recombinants elicited robust virus neutralizing antibody response in pigs. Notably, although both recombinant viruses elicited IAV-S-specific T-cell responses, the frequency of IAV-S specific proliferating T cells secreting IFN- $\gamma$ upon re-stimulation was higher in OV-HA-NPimmunized animals than in the OV-HA group. Importantly, IgG1/IgG2 isotype ELISAs revealed that immunization with OV-HA induced Th2-biased immune responses, whereas immunization with OV-HANP virus resulted in a Th1-biased immune response. While pigs immunized with either OV-HA or OV-HANP were protected when compared to non-immunized controls, immunization with OV-HA-NP resulted in better protective efficacy as evidenced by reduced virus shedding in nasal secretions and reduced viral load in the lung. This study demonstrates the potential of ORFV-based vector for control of swine influenza virus in swine.

Key words: Orf virus, swine influenza virus, vectored-vaccine, neutralizing antibodies, cell-mediated

44 immunity

Effective influenza A virus (IAV-S) vaccines capable of providing robust protection to genetically diverse IAV-S in swine are lacking. Here, we explored the potential of orf virus based vectors expressing the hemagglutining (HA) or both the HA and the nucleoprotein (NP) genes of influena $A$ virus (IAV-S) in eliciting protection against IAV-S in pigs. We observed that both recombinant viruses elicited IAV-Sspecific humoral and cell-mediated immune responses in pigs. Addition of the NP and co-expression of

51 this protein with $\mathrm{HA}$, another major influenza protective antigen, resulted in higher $\mathrm{T}$ cell responses

52 which presumably led to better protection in OV-HA-NP immunized animals, as evidenced by lower

53 levels of virus shedding and viral load in lungs. This study highlights the the potential of ORFV as a vector

54 platform for vaccine delivery against IAV-S. Results here provide the foundation for future development 55 of broadly protective ORFV-based vectors for IAV-S for use in swine. 


\section{Introduction}

Swine influenza is a highly contagious respiratory disease of pigs caused by influenza A viruses in swine (IAV-S). IAV-S is an enveloped, single stranded RNA virus of the family Orthomyxoviridae. The IAVS genome consists of eight single-stranded negative-sense RNA segments encoding four structural (HA, NA, NP and M) and four non-structural (PB1, PB2, PA and NS) proteins. Influenza viruses are classified into subtypes based on the antigenicity of hemagglutinin (HA) and neuraminidase (NA) proteins present on the surface of the virus. There are three recognized subtypes of IAV-S that are currently circulating in the US: H1N1, H1N2 and H3N2 (1). The H1N1 subtype is the major subtype that has been prevalent in the US swine population for several decades; however, recent epidemiological data suggests an increasing incidence of H1N2 and H3N2 IAV-S subtypes $(2,3)$. IAV-S causes acute respiratory disease in pigs resulting in high morbidity (up to $100 \%$ ). The mortality rate is usually low (1-4\%) with most infected animals recovering within 3-7 days of infection $(4,5)$. The median yearly herd prevalence of IAV-S reported in the US is approximately $28 \%$, but it can reach up to $57 \%$ in winter and spring months (6). IAV-S results in significant economic losses to the swine industry mainly due to weight loss, increased time to market, costs associated with treatment of secondary bacterial infections and mortality. This makes IAV-S one of the top three health challenges to the swine industry affecting pigs in all phases of production $(7,8)$. In addition to IAV-S, pigs are also susceptible to infection with avian and human IAVs thereby providing a niche for genetic reassortment between avian/human or swine influenza viruses. This poses a major threat for emergence of new subtypes as well as increases the risk of zoonotic transmission of IAVs. Therefore, effective prevention and control measures for IAV infections in swine have direct impacts on both animal and human health.

Currently, most available IAV-S vaccines are based on whole inactivated virus (WIV). However, these vaccines have not been able to effectively control IAV in swine and in some cases vaccine associated enhanced respiratory disease has been observed when there is an antigenic mismatch between vaccine strain and infecting strain (9). A live-attenuated influenza virus (LAIV) vaccine based on a virus containing a deletion of the NS1 gene, has been recently licensed for use in pigs in the US and may overcome some of the drawbacks of WIV vaccines (10). However, LAIV vaccines have the potential to reassort with the endemic viruses potentially resulting in new influenza virus variants. Indeed, novel variants that arose from reassortment between the vaccine virus and endemic field strains have been recently reported (11). These observations highlight the need for safer and more efficaceous IAV-S 
vaccine candidates. Here we investigated the potential of vectored vaccine candidates based on the parapox orf virus (ORFV) in controlling IAV-S infection in pigs.

Orf virus (ORFV) belongs to genus Parapoxvirus within the family Poxviridae (12) and is a ubiquitous virus that primarily causes a self-limiting mucocutaneous infection in sheep, goats and wild ruminants $(13,14)$. ORFV contains a double-stranded DNA genome with approximately $138 \mathrm{kbp}$ in length and encodes 131 putative genes, including several with immunomodulatory (IMP) functions (15). Given ORFV IMP properties, the virus has long been used as a preventive and therapeutic agent in veterinary medicine $(16,17)$. Additionally, the potential of ORFV as a vaccine delivery platform against several viral diseases in permissive and non-permissive animal species has been explored by us and others (18-24). ORFV based vectored-vaccine candidates have been shown to induce protective immunity against pseudorabies virus (PRV), classical swine fever virus (CSFV) and porcine epidemic diarrhea virus (PEDV) $(22,25-27)$. Among the features that make ORFV a promising viral vector for vaccine delivery in swine are : (i) its restricted host range, (ii) its ability to induce both humoral and cellular immune response $(22,28)$, (iii) its tropism which is restricted to skin keratinocytes with no evidence of systemic dissemination, (iv) lack of vector-specific neutralizing antibodies which allows efficient prime-boost strategies using the same vector constructs $(29,30)$, and $(v)$ its large genome size with the presence of several non-essential genes, which can be manipulated without severely impacting virus replication. Additionally, ORFV encodes several genes with well-characterized immunomodulatory properties. These include a homologue of interleukin 10 (IL-10) (31), a chemokine binding protein (CBP) (32), an inhibitor of granulocyte-monocyte colony stimulating factor GMC-CSF) (33), an interferon resistance gene (VIR) (34), a homologue of vascular endothelial growth factor (VGEF) (35), and inhibitors of nuclear-factor kappa-B (NF-kB) signaling pathway (36-39). The presence of these well-characterized immunomodulatory proteins allowed us to rationally engineer ORFV-based vectors with enhanced safety and immunogenicity profile for use in livestock species, including swine (22-24).

Here we assessed the immunogenicity and protective efficacy of recombinant ORFV vectors expressing the HA protein alone or the HA and the nucleoprotein (NP) of IAV-S. While the HA protein contains immunodominant epitopes recognized by neutralizing antibodies $(40,41)$, the NP protein contains highly conserved immunodominant T-cell epitopes (42). We investigated whether coexpression of HA and NP would enhance the protective efficacy against IAV-S following intranasal challenge infection in pigs.

\section{Results}


117 Construction of ORFV recombinants. The OV-HA recombinant virus was obtained by inserting the full-

118 length HA gene of IAV-S (H1N1) into ORFV121 locus by homologous recombination between a transfer 119 plasmid pUC57-121LR-SIV-HA-loxp-GFP and the parental ORFV strain IA82 (Fig 1A). The OV-HA-NP recombinant virus was obtained by inserting the full-length HA gene into ORFV121 locus and the NP

121 gene into ORFV127 locus. The wild type ORFV strain IA82 was used to generate the OV-HA virus which

122 served as a parental virus for generation of the OV-HA-NP recombinant (Fig 1B). Expression of HA was

123 driven by the vaccina virus (VACV) I1L promoter (43) and expression of the NP gene was driven by the

124 VACV vv7.5 promoter (44). After infection with the parental virus and transfection with the

125 recombination plasmid, the recombinant viruses were obtained and selected. Several rounds of plaque

126 assays were performed to obtain purified recombinant viruses. Once the recombinant viruses were

127 purified and verified by PCR, the marker gene encoding for the green fluorescent protein (GFP) was

128 removed by using the Cre recombinase system. Whole-genome sequencing of plaque purified

129 recombinant viruses was performed after Cre recombinase treatment. Sequencing results confirmed the

130 integrity and identity of ORFV sequences, demonstrated the presence of HA gene, deletion of ORFV121

131 in OV-HA construct, the presence of HA and NP genes and deletion of ORFV121 and ORFV127 genes in

132 OV-HA-NP construct.

Replication kinetics of OV-HA and OV-HA-NP viruses in vitro. Replication properties of both

134 recombinant viruses (OV-HA and OV-HA-NP) were assessed in vitro in primary ovine fetal turbinate cells

135 (OFTu) and primary swine turbinate cells (STU) using one-step and multi-step growth curves (Fig 1C).

136 Cells were infected with an MOI of 0.1 or 10 and cell lysates were harvested at $6,12,24,48,72$ hours

137 post-infection. Both recombinants replicated efficiently in natural host OFTu cells. However, replication

138 of OV-HA and OV-HA-NP viruses was markdely impaired in the STU cells (Fig 1C), which increases the

139 safety profile of the vector for use in pigs.

140 Expression of heterologous proteins by OV-HA and OV-HA-NP recombinant viruses. Expression of the

141 HA protein and NP proteins by OV-HA and/or OV-HA-NP viruses was confirmed by immunofluorescence

142 assay (IFA) and flow-cytometry. As shown in the figure 2A, OV-HA recombinant expressed high levels of

143 HA and OV-HA-NP recombinant expressed high levels of HA and NP proteins (Fig 2A). Expression of HA

144 and NP were also confirmed by flow cytometry (Fig 2C). The IFA was also performed in non-

145 permeabilized cells. Both HA and NP proteins were detected in non-permeabilied cells; however, the

146 levels of protein detected were slightly lower than in permeabilized cells (Fig 2B). As expected this

147 decrease was more evident for NP protein than for the HA protein. These findings suggest that while a 
great proportion of the HA protein expressed by both OV-HA and OV-HA-NP recombinant viruses localizes to the cell surface, and expression of the NP protein is mostly confined to the intracellular compartment.

Immunigenicity of OV-HA and OV-HA-NP in pigs. To assess the immunogenicity of OV-HA and OV-HANP, 4-week old, IAV-S negative, weaned piglets were immunized intramuscularly with two doses of OVHA and OV-HA-NP at a 21 day interval (Fig 3A; Table 1). Antibody response were evaluated using virus neutralization (VN) and hemagglutination inhibition (HI) assays. One week after the first immunization, neutralizing antibodies were detected in both vaccinated groups, however the levels were significantly higher in OV-HA-NP vaccinated animals (Fig 3B). An anamnestic increase in neutralizing antibody titers was seen in both vaccinated groups one week after the boost immunization ( 28 days postimmunization). After the booster immunization all animals maintained high level of neutralizing antibody levels untill the end of the experiment (42 dpi, Fig. 3B).

Serological responses were also measured using an hemagglutination inhibition (HI) assay. The presence of $\mathrm{HI}$ antibodies were detected in OV-HA-NP group on day 7 pi. Similar to the VN results, an anamnestic increase in $\mathrm{HI}$ antibody titers was observed one week after the booster immunization in both groups (Fig $3 \mathrm{C}$ ). Interestingly, the HI titers in the OV-HA group increased significantly after challenge, which is more evident a week after challenge ( $42 \mathrm{dpi}$ ). Such anamnestic increase in $\mathrm{HI}$ titers was not seen in OV-HA-NP-immunized animals, suggesting enhanced protection from IAV-S challenge in this group (Fig 3C). Overall, these results demonstrate that immunization with OV-HA and OV-HA-NP viruses elicited high IAV-S specific neutralizing and $\mathrm{HI}$ antibody responses in immunized pigs.

IAV-S-specific IgG isotype responses elicited by immunization with OV-HA and OV-HA-NP viruses. IAVS specific IgG responses were measured using a whole virus ELISA. Low levels of IAV-S-specific total IgG antibodies were detected in OV-HA and OV-HA-NP immunized groups on 21 days pi (Fig 4A). Similar to $\mathrm{VN}$ and $\mathrm{HI}$ assay, significantly higher levels of IgG antibodies was observed a week following the boost immunization (day 28 pi). Thereafter consistently higher levels of IgG were detected in serum of both OV-HA and OV-HA-NP immunized groups until the end of the experiment (Fig 4A). As expected, expression and delivery of the NP by the OV-HA-NP recombinant virus elicited higher levels of IgG antibodies in immunized pigs after the booster immunization on day 21 pi when compared to those observed in OV-HA-immunized animals ( $P<0.0001$, Fig 4A). 
The endpoint titer of IgG1 and IgG2 isotype antibodies elicited by immunization with OV-HA and OV-HA-NP were determined by an isotype ELISA performed on serum samples collected on 35 days pi. Immunization with OV-HA and OV-HA-NP viruses elicited similar levels of IgG1 response, however, significantly higher titers of IgG2 antibodies were detected in OV-HA-NP-immunized animals when compared to IgG2 titers detected in OV-HA-immunized animals (Fig 4B and 4C). The ratio of Th2associated IgG1 isotype and Th1-associated IgG2 isotype (IgG1/IgG2 ratio) calculated based on the endpoint titers detected in each group was 1.31 (i.e. $>1$ ) for the OV-HA group and 0. 48 ( i.e. $<1$ ) for the OV-HA-NP group (Fig 4D). The IgG1/IgG2 ratio in OV-HA-NP group was significantly lower than in the OVHA group ( $P=0.0048$, Mann-Whitney test). Together these results suggest that the immune response in OV-HA group is mostly Th2 biased. In contrast, the immune response was Th1 biased on the OV-HA-NP group as indicated by higher levels of IgG2 antibodies in the serum of OV-HA-NP immunized animals.

\section{Cellular immune responses elicited by immunization with OV-HA and OV-HA-NP. IAV-S-specific T-cell} responses elicited by immunization with OV-HA and OV-HA-NP viruses was assessed on peripheral blood mononuclear cells (PBMCs) collected on 35 days pi (pre-challenge infection). The frequency of different T-cell subsets secreting IFN-y following re-stimulation with IAV-S was measured using intracellular cytokine staining (ICS) assays. Upon singlet selection, live/dead cell discrimination, IFN-y expression by different T-cell subsets including total T-cells (CD3+), CD4+ T-cells (CD3+/CD4+), CD8+ T-cells (CD3+/CD4CD8+), double positives (CD3+/CD4+/CD8+) and double negative T-cells (CD3+/CD4-/CD8-) were assessed. Animals immunized with either OV-HA or OV-HA-NP had significantly higher percentage of $\mathrm{CD}^{+}$T-cells secreting IFN- $y$ when compared to the non-immunized control animals (Fig 5A). Notably, within the vaccinated animals, OV-HA-NP group presented a significantly higher frequency of IFN-y secreting $\mathrm{CD}^{+} \mathrm{T}$-cells than the OV-HA group $(P=0.0055)$. The animals in the OV-HA-NP group presented higher frequency of IFN-y secreting CD3+/CD4+ T-cells, however, the differences between the groups was not statistically significant. Both immunized groups presented increased frequencies of CD3+/CD8+, CD3+/CD4+/CD8+ (double positives) and CD3+/CD4-/CD8- (double negative) IFN-y secreting T-cell subsets when compared to the control sham-immunized group (Fig 5A).

IAV-S-specific T-cell responses were also evaluated by the carboxyfluorescein succinimidyl ester (CFSE) dilution assay to determine the specific T-cell subsets proliferating upon re-stimulation of PBMCs with inactivated IAV-S. As described above for the IFN-y ICS, upon singlet selection and dead cell exclusion, 206 proliferation by the major swine T-cell subsets was evaluated (Fig 5B). While proliferation of CD3+ T-cell 
208

209

210

211

212

213

214

215

216

217

218

219

220

221

222

223

224

225

226

227

228

229

230

231

232

233

234

235

236

237

238

cells was observed in OV-HA-NP group upon recall stimulation ( $P=0.0095 ;$ Fig 5B). Additionally, a significant increase in the proliferation of CD3+/CD8+ T-cell subset was observed in the OV-HA-NP group $(P=0.0217$, Fig 5B). An increase in proliferation of $C D 3+/ C D 4+T$-cells was also observed (Fig 5B); however, the differences between the treatment groups were not statistically significant (Fig 5B). Overall, these results show that both OV-HA and OV-HA-NP group were able to induce IAV-S-specific Tcell responses in the immunized animals. As expected, T-cell responses elicited by immunization with the OV-HA-NP construct was higher than those observed in animals immunized with the single gene OVHA construct.

Protective efficacy of OV-HA and OV-HA-NP viruses intranasal IAV-S challenge. The protective efficacy of OV-HA and OV-HA-NP were evaluated upon intranasal challenge with IAV-S (after day 35 pi). Virus shedding was assessed in nasal secretions and viral load and pathology were evaluated in the lung. Nasal swabs were collected on days $0,1,3$, and 7 post-challenge (pc) and IAV-S RNA levels were investigated in nasal secretions using real-time reverse transcriptase PCR (rRT-PCR). On day 1 pc, significantly lower IAV-S genome copy numbers - indicating reduced virus shedding - was detected in both OV-HA and OVHA-NP immunized groups when compared to the control sham immunized group (Fig 6A). Only two animals (2/8) in the OV-HA-NP group were positive for viral RNA on day $1 \mathrm{pc}$. On $3 \mathrm{dpc}$, while all animals in control group (8/8) were positive and presented high genome copy numers of IAV-S in nasal secretions, only three animals (3/8) in OV-HA-NP were positive for viral RNA (Fig 6A). Notably, the amount of IAV-S RNA shed by OV-HA-NP-immunized animals were significantly lower than the amount shed by control or OV-HA immunized animals. It is also important to note that animals in OV-HA group had significantly lower level of viral RNA than control group on day 3 pi (Fig. 6A). On day 7 postchallenge, all animals (8/8) in the control sham-immunzied group were still shedding IAV-S in nasal secretions, while only two animals (2/8) in the OV-HA-immunized group were positive presenting low viral RNA copy numbers in nasal secretions. Notably, none of the animals in the OV-HA-NP-immunized group were shedding IAV-S in nasal sercetions on day 7 pi (Fig 6A). These results demonstrate that immunization with OV-HA and OV-HA-NP resulted in decreased virus shedding and shorter duration of virus shedding in nasal secretions following intranasal IAV-S challenge. Notably, these differences were more pronounced in OV-HA-NP-immunized animals.

Shedding of infectious IAV-S was also assessed in nasal secretions collected on days $0,1,3$, and 7 post-challenge. Each sample was subjected to three blind passages in MDCK cells. An immunofluorescence assay using an IAV-S NP-specific monoclonal antibody was performed on the third 
239

240

241

242

243

244

245

246

247

248

249

250

251

252

253

254

255

256

257

258

259

260

261

262

263

264

265

266

267

268

passage to confirm isolation of IAV-S. On day 1 pc, 4 (50\%) animals in the control group were positive for IAV-S, while none of the animals from the OV-HA and OV-HA-NP group were positive on VI (Table 2). On day 3 pc, 7 (87.5\%) animals were positive in the sham-immunized control group; 3 (37.5\%) animals were positive in OV-HA immunized group and 1 (12.5\%) animal was positive in OV-HA-NP-immunized group. Statistical analysis confirmed that there was a significant difference in the number of IAV-S positive animals between control group and OV-HA-NP group on $3 \mathrm{dpc}(\mathrm{P}=0.0101$ Fisher's exact test) (Table 2). IAV-S was not isolated from any of the animals on day 7 post-challenge (Table 2). These results indicate that both OV-HA and OV-HA-NP recombinants were able to reduce virus replication and shedding in the immunized animals. Importantly, detection of infectious virus in only one out of eight animals in OV-HANP groups highlingts the robust protection provided by immunization of pigs this recombinant virus.

Viral load was assessed in the lung of control and immunized pigs on day $7 \mathrm{dpc}$ by using rRT-PCR. While high amounts of IAV-S RNA were detected in the lung of animals in the control sham-immunized group, immunization with OV-HA or OV-HA-NP led to a marked decrease in viral load in the lung (Fig 6B). Notably, only one animal (1/8) in the OV-HA-NP group and two animals (2/8) in OV-HA group presented IAV-S RNA in lung, whereas all the animals in control group (8/8) were positive for IAV-S RNA.

Significantly lower IAV-S RNA loads were detected in the lung of immunized animals when compared to control animals (Fig 6B)

In addition to viral loads pathological changes were also evaluated in the lung of all animals in the study. At necropsy, macroscopic lesions in the lung were characterized by a pathologist who was blinded to the experimental groups. A summary of the gross lung lesions is provided on Table 3. All animals in the control group presented characteristic plum-colored consolidated areas mostly on the cranioventral areas and interstitial pneumonia. Mild lobular consolidation and interstitial pneumonia was present in 2 animals in OV-HA group and 2 animals in OV-HA-NP group. As expected, the lesions were primarily observed in animals having relatively lower levels of neutralizing antibody titers (Table 3). No microscopic lesions were observed in any animals on day 7 post-challenge. Together these results indicate that immunization with ORFV-based vectors, especially with OV-HA-NP virus provided good protection against intranasal homologous IAV-S challenge in pigs.

\section{Discussion}

In this study we explored the potential of ORFV recombinants expressing the HA or both HA and NP proteins of IAV-S in providing protection against intranasal challenge infection in swine. Previous 
work from our group have shown that rational vector design by deleting well-characterized immunomodulatory genes of ORFV is useful in developing highly effective vaccine delivery platforms

271 resulting in safe and highly immunogenic vaccine candidates. One of the well characterized ORFV IMPs is

272 ORFV121, which encodes an NF-KB inhibitor that determines ORFV virulence and pathogenesis in the

273 natural host (39). We have developed highly immunogenic vaccine candidates for porcine epidemic

274 diarrhea virus (PEDV) and rabies virus (RabV) by inserting appropriate protective antigens (spike glycoprotein for PEDV; rabies glycoprotein for RabV) in the ORFV121 gene locus. Given the immunogenicity and safety profile of the OV-PEDV-S and OV-RABV-G recombinant virus in swine, here we constructed an OV-HA recombinant by inserting the HA gene of IAV-S virus in ORFV121 locus. Moreover, to potentially enhance T-cell immune response elicited by the vaccine we generated a second recombinant virus expressing both IAV-S NP and HA proteins. For this, another well-characterized ORFV IMP, the ORFV127 was selected as an insertion site for the NP gene. ORFV127 encodes a viral IL-10 homolog $(15,45)$, which is known to have anti-inflammatory and immunosuppressive activities that may favor immune evasion of the orf virus $(46,47)$. Most importantly, the protein encoded by ORFV127 is known to contribute to ORFV virulence in the natural host (48). Using this approach we tested the hypothesis that simultaneous deletion of two ORFV IMP genes ORFV121 and ORFV127 and concurrent insertion of two highly immunogenic protective antigens of IAV-S (HA and NP) would enhance the immunogenicity of the recombinant virus in swine and provide higher protective efficacy from IAV-S challenge. While the data presented here show that both recombinants OV-HA and OV-HA-NP induced robust immune response against IAV-S in pigs, the immunogenicity and protective efficacy of OV-HA-NP was indeed higher than that elicited by the OV-HA recombinant which substantiates our hypothesis. The increased protective efficacy seem to be related to stronger T-cell response elicited by immunization with the OV-HA-NP virus.

Following challenge infection, we observed interesting differences in the antibody response elicited by OV-HA and OV-HA-NP immunization. As expected, intranasal challenge with IAV-S in sham

294 immunized pigs resulted in anamnestic VN response. Notably, in the immunized groups the VN antibody 295 titers increased by a greater magnitude in the OV-HA group than in the OV-HA-NP group. In the OV-HA 296 group, the geometric mean VN titers were 380.5 and 3319.9 on days 0 and 7 pc respectively, indicating a 297 9-fold increase in the VN titer after challenge. Whereas in OV-HA-NP group, the geometric mean VN 298 titers were 1395.8 and 1810.19 on days 0 and 7 pc respectively, which is only a modest 1.2-fold increase 299 in VN titers pc. Importantly, the VN titers in OV-HA-NP immunized group increased only in the two 300 animals that had the lowest VN titer on day $0 \mathrm{pc}$. The VN titers in the remaining 6 animals in the OV-HA- 
301 NP group remained constant following challenge infection. These results demonstrate that

302 immunization with the OV-HA-NP recombinant virus provided robust immune protection against

303 intranasal IAV-S challenge, with most animals not seroconverting to the challenge virus.

The importance of T-cells in influenza virus clearance and their cross-reactive potential has been well documented $(49,50)$. In this context, CD4+ T-cells help with activation, differentiation and antibody production by virus-specific B cells (51). Additionally, CD4+ helper cells also play an important role in CD8+ cytotoxic T cell activation. Activated CD8+ cytotoxic T-cells function in virus clearance by killing infected cells (52). The NP protein of influenza virus is known to contain several immunologically dominant T-cell epitopes and it is the main antigen recognized by cytotoxic T- lymphocytes (CTL) during influenza A virus infections (42, 53-56). The Immune Epitope Database and Analysis Resource, a

311 manually curated database of experimentally characterized immune epitopes, has recorded 248 T-cells

312 epitopes for nucleoprotein (NP) of influenza virus. Given that NP is relatively conserved among influenza 313 viruses, including in IAV-S, this protein has been one of the target viral antigens for the development of 314 universal influenza vaccine candidates. Because of these important immunological properties, we have 315 developed and evaluated the OV-HA-NP construct expressing both HA and NP proteins. We found that cell mediated immune responses were enhanced by co-delivery and expression of IAV-S HA and NP by

317 OV-HA-NP in pigs when compared to OV-HA group. A significantly higher frequency of CD3+ T-cells 318 proliferated and expressed IFN-y upon re-stimulation with IAV-S in the OV-HA-NP-immunized group. 319 Importanly, immunization with OV-HA-NP resulted in a increased frequency of CD3+/CD8+ T cells upon restimulation with IAV-S. While overall T-cell responses were higher in OV-HA-NP group, an increase in

321 T-cell response was also seen in OV-HA group when compared to the sham immunized group, as

322 evidenced by increase in IFN-y secreting CD3+ T-cell population following antigen stimulation. This can

323 be explained by the presence of several T-cells epitopes in the IAV HA protein, the majority of which have been identified as CD4+ T-cell epitopes $(57,58)$. differentiate into Th1- or Th2- helper cells. Th1 cells secrete several cytokines including IFN- $\gamma$ and IL-12 which help in cell mediated immunity, whereas Th2 cells secrete cytokines like IL-4, IL-6 which contribute to antibody mediated immunity $(40,59)$. Importantly, IgG isotype expression is also

329 controlled by the different cytokines $(60,61)$. In pigs, IFN- $\gamma$ enhances production of IgG2 isotype and 330 hence this IgG isotype is considered to be associated with Th1 immune response. On the other hand, 331 cytokines like IL-4, IL-10 induce secretion of IgG1 and are known to be associated with Th2 immune 
332

333

334

335

336

337

338

339

340

341

342

343

344

345

346

347

348

349

350

351

352

353

response (62). Thus, the ratio of $\operatorname{IgG1}: \operatorname{IgG} 2$ can be used to infer Th1/Th2 bias in response to vaccination. In this study, we found a higher level of IgG1 in pigs immunized with OV-HA recombinant (IgG1:IgG2 >1, Th2 bias), which suggests that the protection may have been mostly antibody-mediated in this group. Conversely, in OV-HA-NP group, the levels of $\operatorname{IgG} 2$ were higher (IgG1:IgG2 $<1$, Th1 bias), which suggests a bias towards cell-mediated immunity in this group. Given that NP protein is known to induce cellmediated immunity, it would be safe to assume that this Th1 bias might be due to NP protein present in the OV-HA-NP recombinant.

This study further demonstrates the use of ORFV as a vaccine delivery platform in swine. The study also shows that two ORFV IMP encoding genes (ORFV121 and ORFV127) can be deleted simulateously from the virus genome to efficiently delivery at least two viral antigens in swine. One of the advantages of ORFV-based vectors is that same vector can be used repeatedly for prime-boost regimens. This is important because pre-existing immunity precludes the use of many vector platforms for vaccine delivery. The humoral immune response data presented here shows that a boost effect was induced after second immunization. In fact, previous findings from our lab show that similar effect can be observed even after three immunizations with ORFV. The recombinant HA and NP protein used in this study share $95 \%$ animo acid identity with the HA and NP protein of the challenge virus. In future, we plan to use the HA gene from other IAV-S subtypes to develop multivalent vaccine candidates and evaluate heterosubtypic protection. The analysis of secretory IgA immune response, which play an important role in providing mucosal immune response is lacking in this study. Future studies involving detailed analysis of mucosal immune response elicited by ORFV-based constructs and challenge infection with heterologous IAV strains are warranted. Nonetheless, results presented here demonstrate that ORFV-based vectors can be important tools to develop improved vaccine candidates to effectively control IAV-S infections in swine.

\section{Material and methods}

Cells and viruses. Primary ovine turbinate cells (OFTu), Madin-Darby canine kidney cells (MDCK) and swine turbinate cells (STU) were cultured at $37{ }^{\circ} \mathrm{C}$ with $5 \% \mathrm{CO}_{2}$ in minimum essential medium (MEM) supplemented with $10 \%$ FBS, 2 mM L-glutamine and containing streptomycin (100 $\mu \mathrm{g} / \mathrm{mL})$, penicillin (100 $\mathrm{U} / \mathrm{mL}$ and gentamycin $(50 \mu \mathrm{g} / \mathrm{mL})$.

The ORFV strain IA82 (OV-IA82; kindly provided by Dr. Daniel Rock at University of Illinoir UrbanaChampaign), was used as the parental virus to construct the recombinants and in all the experiments 
362

363

364

365

366

367

368

369

370

371

372

373

374

375

376

377

involving the use of wild-type ORFV. Wild-type and recombinant ORFV viruses were amplified in OFTu cells. Swine influenza virus H1N1 A/Swine/OH/24366/2007 (H1N1), kindly provided by Gourapura Lab was used for virus challenge, virus neutralization assay, hemagglutination inhibition ( $\mathrm{HI})$, and as a coating antigen for whole virus ELISA. The H1N1 A/Swine/OH/24366/2007 (H1N1) virus was propagated in MDCK cells using DMEM containing TPCK-treated trypsin ( $2 \mu \mathrm{g} / \mathrm{mL}$ ) and $25 \mathrm{mM} \mathrm{HEPES}$ buffer.

Generations of recombination plasmids. To insert the heterologous IAV-S gene in the ORFV121 locus, a recombination plasmid containing right and left flanking sequences of the ORFV121 gene were inserted into pUC57 plasmid. The HA gene of swine influneza virus, A/SW/OH/511445/2007 (OH7) (GenBank: EU604689) (63) was inserted between the ORFV121 flanking sequence in the pUC57 plasmid. The HA gene was condon optimized for swine species (GenScript). The HA gene was cloned under the vaccinia virus (VACV) I1L promoter (5'-TATTTAAAAGTTGTTTGGTGAACTTAAATGG - 3' ) (43) and a flag-tag epitope (DYKDDDK) was fused to the amino terminus of the HA gene to detect its expression. The gene encoding green fluorescent protein (GFP) was inserted downstream of HA gene and used as a selection marker for recombinant virus purification. The GFP sequence was flanked by loxp sequences $5^{\prime}$ -

ATAACTTCGTATAATGTATACTATACGAAGTTAT-3' to allow for removal of GFP by Cre recombinase following recombinant virus purification. This recombination cassette was named pUC57-121LR-SIV-HA$\operatorname{loxp-GFP}($ Fig 1A).

Similarly, another recombination cassette was generated to insert NP gene of IAV-S into the ORFV ORFV127 locus. A recombination cassette for ORFV127 was constructed as describe above with the ORFV127 left and right flanking regions being cloned into the pUC57-LoxP-GFP plasmid (pUC57-127LRLoxP-GFP. The nucleoprotein (NP) gene of swine influenza virus, A/SW/OH/511445/2007 (OH7) (GenBank: EU604694) (63) was inserted between ORFV127 left and right flanks. The NP gene was cloned under the VACV vv7.5 promoter (44) and the HA epitope tag sequence (YPYDVPDYA) was fused at the amino terminus of the NP protein to detect its expression by the recombinant virus. In addition, an eukaryotic Kozak consensus sequence 5'-gccaaccATGg-3' (64), where ATG refers to the start codon of the NP gene, was added immediately downstream of vv7.5 promoter. This recombination cassette was named pUC57-127LR-SIV-NP-loxp-GFP (Fig 1B).

Generation of recombinant OV-HA and OV-HA-NP viruses. The HA gene of IAV-S was inserted into the ORFV121 locus of the ORFV genome by homologous recombination. Briefly, OFTu cells cultured in 6-well plate were infected with OV-IA82 with a multiplicity of infection (MOI) of 1 . Three hours later, the infected cells were transfected with $2 \mu \mathrm{g}$ of pUC57-121LR-SIV-HA-loxp-GFP using Lipofectamine 3000 
according to the manufacturer's instruction (Invitrogen, catalog no: L3000-075). At 48 hours postinfection/transfection cell cultured were harvested, subjected to three freeze-and-thaw cycles. The ORFV recombinant expressing IAV-S HA was purified using plaque assay by selecting viral foci expressing GFP. After several rounds of plaque purification, the presence of HA gene and absence of ORFV121 gene was confirmed by PCR as described before $(22,24)$ and the insertion and integrity of the whole genome sequence of the recombinant was confirmed sequencing using Nextera XT DNA library preparation following by sequencing on the Illumina Mi-Seq sequencing platform. Once the purified recombinant virus was obtained, the GFP selection gene was removed by using Cre recombinase treatment as described below. This recombinant is referred to as OV-HA throughout this manuscript.

Similarly, double gene expression vector containing the IAV-S HA and NP genes in ORFV121 and the ORFV127 gene loci (48), respectively was generated by homologous recombination. Both ORFV121 and ORFV127 are virulence determinants that contribute to ORFV IA-82 virulence in the natural host $(39,48)$. For this, infection/transfection was performed by infecting OFTu cells with the OV-HA recombinant virus and transfecting with pUC57-127LR-SIV-NP-loxp-GFP plasmid. The recombinant virus was purified using plaque assay as described above and following purification the GFP reporter gene was removed using the Cre recombinase treatment described below. The resulting recombinant ORFV vector expressing the HA and NP gene is referred to as OV-HA-NP in this manuscript.

The Cre/loxP recombination system was used to remove the GFP reporter gene from the OV-HA or OV-HA-NP recombinants. A plasmid pBS185 CMV-Cre, carrying the cre gene under the hCMV promoter was a kind gift from Brian Sauer (65) (Addgene catalog number : 11916). OFTu cells were plated in a 24- well plate and 24h later transfected with $500 \mathrm{ng}$ of the pBS185-CMV-Cre plasmid using Lipofectamine 3000 (Invitrogen, catalog num: L3000-075) according to the manufacturer's instructions. Approximately $24 \mathrm{~h}$ after transfrections, cells were infected with $\sim 1 \mathrm{MOI}$ of the plaque purified recombinant viruses (OV-HA-GFP or OV-HA-NP-GFP). Approximately $48 \mathrm{~h}$ post-infection, the cre recombinase treated recombinant viruses were harvested and subjected to a second round of Cre treatment as described above. Following cre recombinase treatment, two to three rounds of plaque assays were performed to select foci lacking GFP expression and to obtain reporterless OV-HA or OV-HANP recombinant viruses. Following markerless virus selection complete genome sequencing was performed to determine the integrity of ORFV and IAV-S sequences in the recombinant OV-HA and OVHA-NP viruses. 
Growth curves. Replication kinetics of OV-HA and OV-HA-NP recombinant viruses were assessed in vitro

424 in OFTu and STU cells. Briefly, OFTu and STU cells cultured in 12-well plates were inoculated with OV-HA or OV-HA-NP with a multiplicity of infection (MOI) of 0.1 (multistep growth curve) or 10 (single-step growth curve) and harvested at 6, 12, 24, 48, 72 hours post-infection (hpi). Virus titers in cell lysates and supernatants were determined on each time point using Sperman and Karber's method and expressed

428 as tissue culture infectious dose $50\left(\mathrm{TCID}_{50}\right)$ per milliliter $(66)$.

Immunofluorescence. Immunofluorescence assay (IFA) was used to assess expression of the heterologous proteins by the OV-HA or the OV-HA-NP viruses as described previously (67). Briefly, OFTu cells were inoculated with each recombinant virus ( $\mathrm{MOI}$ of 1 ) and fixed with $3.7 \%$ formaldehyde at 48 hours pi. Then, cells were permeabilized with $0.2 \%$ PBS-Triton X-100 for $10 \mathrm{~min}$ at room temperature. Another set of samples which were not permealized were also tested side-by-side to compare the expression pattern between permeabilized and non-permeabilied cells. Flag-tag specific mouse antibody

435 (Genscript, catalog no: A100187) and HA-tag specific rabbit antibody (Cell Signaling, catalog no: 3724S)

436 were used as primary antibody to detect HA and NP protein respectively. Then, cells were incubated 437 with Alexa fluor 594 goat anti-mouse IgG $(\mathrm{H}+\mathrm{L})$ secondary antibody (Invitrogen, catalog no: A11005) or 438 Alexa fluor 488 goat anti-rabbit IgG antibody and cells were observed under fluorescence microscope.

439 Animal immunization and challenge studies. The immunogenicity of the two recombinant viruses (OV440 HA and OV-HA-NP) was evaluated in 3-week old high-health pigs. A summary of experimental design is 441 presented in Table 1. Twenty-four pigs, seronegative for IAV-S, were randomly allocated into three 442 experimental groups as follows: Group 1, sham immunized ( $n=8)$; Group 2, OV-HA immunized $(n=8)$;

443 Group 3, OV-HA-NP immunized $(\mathrm{n}=8)$. Immunization was performed by intramuscular injection of $2 \mathrm{ml}$ of 444 a virus suspension containing $10^{7} \mathrm{TCID}_{50} / \mathrm{mL}$ in MEM. All animals were immunized on day 0 and received 445 a booster immunization on day 21 post-immunization. All animals were challenged intranasally on day 44635 post-immunization with $5 \mathrm{~mL}$ virus inoculum containing $6 \times 10^{6} \mathrm{TCID}_{50}$ of H1N1 447 A/Swine/OH/24366/2007 (H1N1) (68) per animal. Animals were monitored daily for clinical signs of IAV448 S. Serum and PBMC samples were collected on days $0,7,14,21,28,35,38$ and 42 days post449 immunization. Nasal swabs were collected on days $0,1,3,7$ post-challenge. The experiment was 450 terminated on day 42 post-immunization or 7 days post-challenge. Whole lung as a unit were collected 451 from euthanized animals during necropsy and examined grossly for pathologic changes by a pathologist 452 blided to study groups. Animal immunization challenge studies were conducted at South Dakota State 
University (SDSU) Animal Resource Wing (ARW), following the guidelines and protocols approved by the SDSU Institutional Animal Care and Use Committee (IACUC approval no. 17-018A)

Virus neutralization (VN) assay. Virus neutralization titer in the serum samples were determined as described previously (69). Briefly, serum samples were heat inactivated for 30 minutes at $56{ }^{\circ} \mathrm{C}$. Two-

457 fold serial dilutions of serum were incubated with 200 TCID $_{50}$ of IAV-S, A/Swine/OH/24366/2007 (H1N1),

458 at $37^{\circ} \mathrm{C}$ for 1 hour. This virus-serum complex was then transferred to a 96 -well plate pre-seeded with

459 MDCK cells $24 \mathrm{~h}$ earlier. After 1 hour of adsorption, virus-serum complex was removed and fresh DMEM

460 containing $2 \mu \mathrm{g} / \mathrm{mL}$ of TPCK-treated trypsin was added to the cells. After 48 -hour incubation at $37^{\circ} \mathrm{C}$,

461 cells were fixed with $80 \%$ acetone. Virus positive MDCK cells were detected by immunofluorescence

462 assay using a mouse monoclonal antibody targeting nucleoprotein (NP) of influenza virus (IAV-NP HB-65

$463 \mathrm{mAb}$; kindly provided by Drs. Eric Nelson and Steve Lawson at SDSU). The virus neutralization titer was

464 defined as the reciprocal of the highest dilution of serum where there was complete inhibition of

465 infection/replication as evidenced by absence of fluorescent foci. Appropriate positive and negative

466 control samples were included in all the plates.

467 Hemagglutination inhibition (HI) assay. $\mathrm{HI}$ assay was performed according to the method descrined

468 previously (69).Briefly, serial 2-fold dilution (starting dilution 1:4) were prepared in PBS. Then 4 HA units

469 of $\mathrm{H} 1 \mathrm{~N} 1 \mathrm{~A} / \mathrm{S}$ wine/OH/24366/2007 virus was added to the serum dilutions and incubated at room

470 temperature for 1 hour. A solution (in PBS) of turkey red blood cells (containing $0.5 \%$ RBC) were added

471 to the wells and allowed to settle. The HI titer was calculated as the reciprocal of the highest dilution of

472 sera that inhibited hemagglutination of turkey RBC.

473 Real-time reverse transcriptase PCR (rRT-PCR). Virus shedding in nasasl secretions and viral load in

474 lungs was evaluated by rRT-PCR. Lung tissues were homogenized using tissue homogenizer by adding 10

$475 \mathrm{~mL}$ of DMEM in $1 \mathrm{~g}$ of lung tissue. Viral nucleic acid was extracted from the nasal swabs and lung tissue

476 homogenates using the MagMax Viral RNA/DNA isolation Kit (Life Technologies). The rRT-PCR tests were

477 performed at Animal Disease Research and Diagnostic Lab (ADRDL), SDSU, SD. Genome copy numbers

478 per milliliter were determined based on the relative standard curve derived from four-parameter logistic

479 regression analysis ( $R$-square $=0.9928$, Root mean square error $(R M S E)=1.0012$ ).

480 Virus isolation. Virus isolation was performed on the nasal swabs collected on day $0,1,3$, and 7 post481 challenge. Nasal swabs were filtered through a 0.22-micron filter and mixed with DMEM containing 2 $482 \mu \mathrm{g} / \mathrm{mL}$ of TPCK-treated trypsin in 1:1 ratio. Then, $250 \mu \mathrm{L}$ of this inoculum was added to 24 -well plate 
containing MDCK cells. The cells were incubated for 1 hour at $37^{\circ} \mathrm{C}$. After 1 hour adsorption, $250 \mu \mathrm{L}$ of DMEM was added to the wells and plate was incubated for 48 hours. After 48 hours, cell lysate was harvested, and two more blind serial passages were performed. After the third passage, the supernatant was collected, and the cells were fixed with $80 \%$ acetone. Immunofluorescence assay (IFA) was performed using IAV-NP mAb (IAV-NP HB-65) as primary antibody and Alexa fluor 594 goat anti-mouse antibody as secondary antibody (Invitrogen, catalog no: A11005). SIV infected cells were identified based on the presence of fluorescent foci.

ELISA. IAV-S-specific IgG, IgG1a and IgG2a immune response elicited by immunization with OV-HA or OV-HA-NP were assessed by whole virus ELISA. The antigen for coating the ELISA plates was prepared as described previosly (70) with some modifications. Briefly, ultra-centrifugation of virus culuture supernatant and the virus pellet in $30 \%$ sucrose cushion gradient were performed using Optima-L 100K

494 ultracentrifuge (Beckman Coulter) at 18,000 RPM for 1.5 hours. The virus pellet was resuspended in 495 DMEM and UV inactivation of the virus was carried out using CL1000 UV crosslinker. Determination of 496 the optimal coating antigen concentration and dilution of secondary antibodies were carried out by checkerboard titration.

To detect IAV-S specific total IgG, Immulon 1B ELISA plates (ThermoFisher Scientific, catalog no: 3355) were coated with $250 \mathrm{ng} /$ well of concentrated and UV inactivated IAV-S virus and incubated at 37 ${ }^{\circ} \mathrm{C}$ for 2 hours. Then plates were washed three times with PBST (1X PBS with 0.5\% Tween-20) and 501 blocked with $200 \mu \mathrm{L} /$ well of blocking solution ( $5 \%$ milk in PBST) and incubated overnight at $4{ }^{\circ} \mathrm{C}$. Then, 502 the plates were washed three times with PBST. Serum samples diluted in blocking solution at the 503 dilution of 1:100 was added, and the plates were incubated for $1 \mathrm{hr}$ at room temperature (RT). After, 504 three washes with PBST, $100 \mu \mathrm{L}$ of biotinylated anti-pig IgG antibody (Bethyl, catalog no: A100-104) 505 diluted in blocking buffer (1:4000) was added to the plate and incubated for $1 \mathrm{hr}$ at RT. Following three 506 washes, HRP-conjugated streptavidin (Thermo Scientific, catalog no: 21136) diluted in blocking solution 507 (1:4000) was added to plates and incubated for $1 \mathrm{hr}$ at RT. Plates were washed again for three times 508 with PBST and $100 \mu \mathrm{L} /$ well of 3,3',5,5'-tetramethylbenzidine (TMB) substrate was added to the plates 509 (KPL, catalog no: 5120-0047). Finally, the colorimetric reaction was stopped by adding $100 \mu \mathrm{L} 1 \mathrm{~N} \mathrm{HCl}$ 510 solution per well. Optical density (OD) values were measured at $450 \mathrm{~nm}$ using a microplate reader. Cut511 off value was determined as mean OD of negative serum samples plus three times of standard deviation 512 (mean + 3SD). 
Isotype ELISA were performed on the serum samples collected on day 35 post-immunization.

514 For isotype ELISA, mouse anti-pig IgG1 (Biorad, catalog no: MCA635GA) and mouse anti-pig IgG2

515 antibody (Biorad, catalog no: MCA636GA) were used as secondary antibodies and plates were incubated

516 with biotinylated anti-mouse antibody (KPL, catalog no: 5260-0048) before incubating with streptavidin-

517 HRP antibody. Endpoint titer ELISA using serial two-fold serial dilutions of serum samples were

518 performed to determine endpoint titer of SIV-specific IgG1 and IgG2 antibody levels in the serum

519 samples. Other procedures were similar to the total IgG ELISA as described above.

520 Flow-cytometry. IAV-S-specific T-cell response elicited by ORFV recombinants was evaluated by an

521 intracellular cytokine staining (ICS) assay for interferon gamma (IFN-y) and T-cell proliferation assay. For

522 IFN-y expression assay, cryopreserved PBMCs collected on day 35 post-immunization (0 dpc) were

523 thawed and seeded at a density of $5 \times 10^{5}$ cells/well in 96 -well plate. Cells were stimulated with UV

524 inactivated IAV-S at MOI of 1. Additionally, cells were stimulated with concanavalin (ConA: $2 \mu \mathrm{g} / \mathrm{ml}$ )

525 (Sigma, catalog no: C0412) plus phytohemagglutinin (PHA: $5 \mu \mathrm{g} / \mathrm{ml}$ ) (Sigma, catalog no: 61764) as

526 positive control and cRPMI (RPMI with 10\% FBS) was added to the negative control wells. Protein

527 transport inhibitor, Brefeldin A (BD Biosciences, catalog no: 555029), was added 6 hours after

528 stimulation and the cells were incubated for 12 hours prior to flow cytometric analysis. For the

529 proliferation assay, PBMCs (35 dpi) were stained with $2.5 \mu \mathrm{M}$ carboxyfluorescin succinimidyl ester (CFSE;

530 in PBS) (BD Horizon, catalog no: 565082). CFSE stained cells were seeded at a density of $5 \times 10^{5}$

531 cells/well in 96-well plate. The cells were stimulated as described above. After stimulation, the cells

532 were incubated for 4 days at $37^{\circ} \mathrm{C}$ with $5 \% \mathrm{CO}_{2}$ prior to staining. Antibodies used for immunostaning the

533 cells were : CD3+ (Mouse anti-pig CD3E Alexa Fluor 647; BD Pharmingen, catalog no: 561476), CD4+

534 (Primary antibody: Mouse anti-pig CD4, Monoclonal Antibody Center (WSU), catalog no: 74-12-4;

535 secondary antibody: Goat anti-mouse IgG2b PE/Cy7, Southern Biotech, catalog no: 1090-17), CD8+

536 (Primary antibody: Mouse anti-pig CD8 $\alpha$, Monoclonal Antibody Center (WSU), catalog no: 76-2-11;

537 secondary antibody: Goat anti-mouse IgG2a FitC, Southern Biotech, catalog no: 1080-02), IFN-y (Anti-pig

538 IFN-y PE, BD Pharmingen, catalog no: 559812. The stained cells were analyzed using Attune NxT flow-

539 cytometer. Results were corrected for background proliferation by subtracting mock-stimulated

540 proliferation from the frequency of cells that responded under inactivated SIV stimulation. The

541 percentage of responding cells was calculated as the percentage of total T cells (live CD3+ cells).

\section{Statistical analysis}


Statistical analysis was performed using Graphpad Prism software. The normality of the data was tested using Shapiro-Wilk test. Comparison of means between the groups was done using two-way ANOVA for normal data or Kruskal Wallis test for non-normal data. Pairwise comparison was done using Tukey multiple comparison test. P value of less than 0.05 was considered significant. Flow cytometry data was analyzed using Flow Jo software.

\section{Acknowldgement}

We thank the Animal Resource Wing (ARW) SDSU for their assistance in animal experiments. We thank the Cornell BRC Flow Cytometry Facility at the Cornell Institute of Biotechnology for the use of flow cytometers for data acquisition. We also would like to thank Bishwas Sharma, Maureen Hoch Vieira Fernandes, Jessica Caroline Gomes Noll, Gabriela Mansano do Nascimento, and Steve Lawson for their help with sample collection. This work was supported by AFRI Foundational and Applied Science Program (grant no. 2017-67015-32034/project accession no. NYCV478904) from the USDA National Institute of Food and Agriculture.

\section{References}

1. Ma W. 2020. Swine influenza virus: Current status and challenge. Virus Res. Elsevier B.V.

2. Anderson TK, Chang J, Arendsee ZW, Venkatesh D, Souza CK, Kimble JB, Lewis NS, Davis CT, Vincent AL. 2020. Swine Influenza A Viruses and the Tangled Relationship with Humans. Cold Spring Harb Perspect Med a038737.

3. USDA. 2020. Influenza A Virus in Swine Surveillance.

4. OIE. 2009. SWINE INFLUENZA.

5. Vincent AL, Ma W, Lager KM, Janke BH, Richt JA. 2008. Swine Influenza Viruses. A North American Perspective. Adv Virus Res. Academic Press.

6. Chamba Pardo FO, Alba-Casals A, Nerem J, Morrison RB, Puig P, Torremorell M. 2017. Influenza herd-level prevalence and seasonality in breed-to-wean pig farms in the Midwestern United States. Front Vet Sci 4:11.

7. CFSH. 2016. Swine influenza.

8. Vincent AL, Perez DR, Rajao D, Anderson TK, Abente EJ, Walia RR, Lewis NS. 2017. Influenza A virus vaccines for swine. Vet Microbiol 206:35-44. 
571 9. Vincent AL, Lager KM, Janke BH, Gramer MR, Richt JA. 2008. Failure of protection and enhanced 572 pneumonia with a US H1N2 swine influenza virus in pigs vaccinated with an inactivated classical swine H1N1 vaccine. Vet Microbiol 126:310-323.

574

575

576

577

578

579

580

10. Genzow M, Goodell C, Kaiser TJ, Johnson W, Eichmeyer M. 2018. Live attenuated influenza virus vaccine reduces virus shedding of newborn piglets in the presence of maternal antibody. Influenza Other Respi Viruses 12:353-359.

11. Sharma A, Zeller MA, Li G, Harmon KM, Zhang J, Hoang H, Anderson TK, Vincent AL, Gauger PC. 2020. Detection of live attenuated influenza vaccine virus and evidence of reassortment in the U.S. swine population. J Vet Diagnostic Investig 32:301-311.

12. ICTV. 2017. Virus taxonomy: Online (10th) Report of the International Committee on Taxonomy of Viruses.

13. Spyrou V, Valiakos G. 2015. Orf virus infection in sheep or goats. Vet Microbiol 181:178-182.

14. Haig DM, Mercer AA. 1998. Ovine diseases. Orf. Vet Res 29:311-26.

15. Delhon G, Tulman ER, Afonso CL, Lu Z, de la Concha-Bermejillo A, Lehmkuhl HD, Piccone ME, Kutish GF, Rock DL. 2004. Genomes of the Parapoxviruses Orf Virus and Bovine Papular Stomatitis Virus. J Virol 78:168-177.

16. Weber O, Knolle P, Volk H-D, Weber, Olaf, Knolle, Percy, Volk H-D, Weber O, Knolle P, Volk H-D. 2007. Immunomodulation by inactivated Orf virus (ORFV) - therapeutic potential, p. 297-310. In Mercer, Andrew, Schmidt, Axel, Weber, O (ed.), PoxvirusesFirst. Birkhäuser Basel, Basel.

17. Weber O, Mercer AA, Friebe A, Knolle P, Volk H-D. 2013. Therapeutic immunomodulation using a virus--the potential of inactivated orf virus. Eur J Clin Microbiol Infect Dis 32:451-60.

18. Amann R, Rohde J, Wulle U, Conlee D, Raue R, Martinon O, Rziha H-J. 2012. A New Rabies Vaccine Based on a Recombinant Orf Virus (Parapoxvirus) Expressing the Rabies Virus Glycoprotein. J Virol 87:1618-1630.

19. Henkel M, Planz O, Fischer T, Stitz L, Rziha H-J. 2005. Prevention of virus persistence and protection against immunopathology after Borna disease virus infection of the brain by a novel Orf virus recombinant. J Virol 79:314-25.

20. Rohde J, Amann R, Rziha H-J. 2013. New Orf Virus (Parapoxvirus) Recombinant Expressing H5 
Hemagglutinin Protects Mice against H5N1 and H1N1 Influenza A Virus) New Orf Virus

(Parapoxvirus) Recombinant Expressing H5 Hemagglutinin Protects Mice against H5N1 and H1N1 Influenza A Virus. PLoS One 8:83802.

21. Rohde J, Schirrmeier H, Granzow H, Rziha H-J. 2011. A new recombinant Orf virus (ORFV, Parapoxvirus) protects rabbits against lethal infection with rabbit hemorrhagic disease virus (RHDV). Vaccine 29:9256-64.

22. Hain KS, Joshi LR, Okda F, Nelson J, Singrey A, Lawson S, Martins M, Pillatzki A, Kutish G, Nelson EA, Flores EF, Diel DG. 2016. Immunogenicity of a Recombinant Parapoxvirus Expressing the Spike Protein of Porcine Epidemic Diarrhea Virus. J Gen Virol https://doi.org/10.1099/jgv.0.000586.

23. Joshi LR, Okda FA, Singrey A, Maggioli MF, Faccin TC, Fernandes MHV, Hain KS, Dee S, Bauermann F V., Nelson EA, Diel DG. 2018. Passive immunity to porcine epidemic diarrhea virus following

24. Martins M, Joshi LR, Rodrigues FS, Anziliero D, Frandoloso R, Kutish GF, Rock DL, Weiblen R, Flores EF, Diel DG. 2017. Immunogenicity of ORFV-based vectors expressing the rabies virus glycoprotein in livestock species. Virology2017/09/13. 511:229-239.

25. Dory D, Fischer T, Béven V, Cariolet R, Rziha H-J, Jestin A. 2006. Prime-boost immunization using DNA vaccine and recombinant Orf virus protects pigs against Pseudorabies virus (Herpes suid 1). Vaccine 24:6256-63.

26. Voigt H, Merant C, Wienhold D, Braun A, Hutet E, Le Potier MF, Saalmüller A, Pfaff E, Büttner M.

622 27. Joshi LR, Okda FA, Singrey A, Maggioli MF, Faccin TC, Fernandes MH V, Hain KS, Dee S, Bauermann F V, Nelson EA, Diel DG. 2018. Passive immunity to porcine epidemic diarrhea virus following immunization of pregnant gilts with a recombinant orf virus vector expressing the spike

626 28. Fischer T, Planz O, Stitz L, Rziha H-J. 2003. Novel recombinant parapoxvirus vectors induce protective humoral and cellular immunity against lethal herpesvirus challenge infection in mice. J 
Virol 77:9312-23.

629 29. Haig DM, McInnes CJ. 2002. Immunity and counter-immunity during infection with the parapoxvirus orf virusVirus Research.

631 30. Mercer AA, Yirrell DL, Reid HW, Robinson AJ. 1994. Lack of cross-protection between vaccinia virus and orf virus in hysterectomy-procured, barrier-maintained lambs. Vet Microbiol 41:373382.

31. Fleming SB, Anderson IE, Thomson J, Deane DL, McInnes CJ, McCaughan CA, Mercer AA, Haig DM. 2007. Infection with recombinant orf viruses demonstrates that the viral interleukin-10 is a virulence factor. J Gen Virol 88:1922-7.

637 32. Seet BT, McCaughan CA, Handel TM, Mercer A, Brunetti C, McFadden G, Fleming SB. 2003. Analysis of an orf virus chemokine-binding protein: Shifting ligand specificities among a family of poxvirus viroceptors. Proc Natl Acad Sci U S A 100:15137-15142.

33. Deane D, Mclnnes CJ, Percival A, Wood A, Thomson J, Lear A, Gilray J, Fleming S, Mercer A, Haig D. 2000. Orf virus encodes a novel secreted protein inhibitor of granulocyte-macrophage colonystimulating factor and interleukin-2. J Virol 74:1313-20.

34. McInnes CJ, Wood AR, Mercer AA. 1998. Orf virus encodes a homolog of the vaccinia virus interferon-resistance gene E3L. Virus Genes 17:107-15.

35. Westphal D, Ledgerwood EC, Hibma MH, Fleming SB, Whelan EM, Mercer AA. 2007. A novel Bcl2-like inhibitor of apoptosis is encoded by the parapoxvirus ORF virus. J Virol 81:7178-88.

36. Diel DG, Delhon G, Luo S, Flores EF, Rock DL. 2010. A novel inhibitor of the NF-\{kappa\}B signaling pathway encoded by the parapoxvirus orf virus. J Virol 84:3962-73.

37. Diel DG, Luo S, Delhon G, Peng Y, Flores EF, Rock DL. 2011. A nuclear inhibitor of NF-kappaB encoded by a poxvirus. J Virol 85:264-75.

38. Khatiwada S, Delhon G, Nagendraprabhu P, Chaulagain S, Luo S, Diel DG, Flores EF, Rock DL. 2017. A parapoxviral virion protein inhibits NF-KB signaling early in infection. PLOS Pathog 13:e1006561. inhibitor of NF-kappaB that contributes to virus virulence. J Virol 85:2037-2049. 
40. Stanekov Z, Varekov E. 2010. Conserved epitopes of influenza A virus inducing protective immunity and their prospects for universal vaccine development. Virol J. BioMed Central.

41. Knossow M, Gaudier M, Douglas A, Barrère B, Bizebard T, Barbey C, Gigant B, Skehel JJ. 2002. Mechanism of neutralization of influenza virus infectivity by antibodies. Virology 302:294-298.

42. Grant E, Wu C, Chan KF, Eckle S, Bharadwaj M, Zou QM, Kedzierska K, Chen W. 2013. Nucleoprotein of influenza A virus is a major target of immunodominant CD8 + T-cell responses. Immunol Cell Biol 91:184-194.

43. Liu X, Kremer M, Broyles SS. 2004. A natural vaccinia virus promoter with exceptional capacity to direct protein synthesis. J Virol Methods 122:141-145.

44. Chakrabarti S, Sisler JR, Moss B. 1997. Compact, synthetic, vaccinia virus early/late promoter for protein expression. Biotechniques 23:1094-1097.

45. Fleming SB, McCaughan CA, Andrews AE, Nash AD, Mercer AA. 1997. A homolog of interleukin-10 is encoded by the poxvirus orf virus. J Virol 71:4857-61.

46. Dumont FJ. 2003. Therapeutic potential of IL-10 and its viral homologues: an update. Expert Opin Ther Pat 13.

47. Moore KW, de Waal Malefyt R, Coffman RL, O'Garra A. 2001. Interleukin-10 and the Interleukin10 Receptor. Annu Rev Immunol 19:683-765.

48. Martins M, Rodrigues FS, Joshi LR, Jos' J, Jardim JC, Flores MM, Weiblen R, Flores EF, Diel DG, Jardim JJ. 2021. Orf virus ORFV112, ORFV117 and ORFV127 contribute to ORFV IA82 virulence in sheep. Vet Microbioogy https://doi.org/10.1016/j.vetmic.2021.109066.

49. Topham DJ, Tripp RA, Doherty PC. 1997. CD8+ T cells clear influenza virus by perforin or Fasdependent processes. J Immunol 159.

50. Soema PC, Van Riet E, Kersten G, Amorij JP. 2015. Development of cross-protective influenza A vaccines based on cellular responses. Front Immunol. Frontiers Media S.A.

51. Sun J, Braciale TJ. 2013. Role of T cell immunity in recovery from influenza virus infection. Curr Opin Virol. Elsevier B.V.

52. Hamada H, Bassity E, Flies A, Strutt TM, Garcia-Hernandez M de L, McKinstry KK, Zou T, Swain SL, 
Dutton RW. 2013. Multiple Redundant Effector Mechanisms of CD8 + T Cells Protect against Influenza Infection . J Immunol 190:296-306.

53. Chen L, Zanker D, Xiao K, Wu C, Zou Q, Chen W. 2014. Immunodominant CD4+ T-Cell Responses to Influenza A Virus in Healthy Individuals Focus on Matrix 1 and Nucleoprotein https://doi.org/10.1128/JVI.01631-14.

54. Gao XM, Liew FY, Tite JP. 1989. Identification and characterization of T helper epitopes in the nucleoprotein of influenza A virus. J Immunol 143.

55. Wu C, Zanker D, Valkenburg S, Tan B, Kedzierska K, Ming Zou Q, Doherty PC, Chen W. 2011. Systematic identification of immunodominant CD8 + T-cell responses to influenza A virus in HLAA2 individuals. PNAS 108:9178-9183.

56. Yewdell JW, Bennink JR, Smith GL, Moss B. 1985. Influenza A virus nucleoprotein is a major target antigen for cross-reactive anti-influenza A virus cytotoxic T lymphocytes. Proc Natl Acad Sci U S A 82:1785-1789.

57. Cassotta A, Paparoditis P, Geiger R, Mettu RR, Landry SJ, Donati A, Benevento M, Foglierini M, Lewis DJM, Lanzavecchia A, Sallusto F. 2020. Deciphering and predicting CD4+ T cell immunodominance of influenza virus hemagglutinin. J Exp Med 217.

58. Bui HH, Peters B, Assarsson E, Mbawuike I, Sette A. 2007. Ab and T cell epitopes of influenza A virus, knowledge and opportunities. Proc Natl Acad Sci U S A 104:246-251.

59. Stevens TL, Bossie A, Sanders VM, Fernandez-Botran R, Coffman RL, Mosmann TR, Vitetta ES. 1988. Regulation of antibody isotype secretion by subsets of antigen-specific helper T cells. Nature 334:255-258.

60. Crawley A, Wilkie BN. 2003. Porcine Ig isotypes: Function and molecular characteristics. Vaccine 21:2911-2922.

61. Bretscher PA. 2014. On the Mechanism Determining the Th1/Th2 Phenotype of an Immune Response, and its Pertinence to Strategies for the Prevention, and Treatment, of Certain Infectious Diseases. Scand J Immunol 79:361-376.

62. Crawley A, Raymond C, Wilkie BN. 2003. Control of immunoglobulin isotype production by porcine B-cells cultured with cytokines. Vet Immunol Immunopathol 91:141-154. 
63. Vincent AL, Swenson SL, Lager KM, Gauger PC, Loiacono C, Zhang Y. 2009. Characterization of an influenza A virus isolated from pigs during an outbreak of respiratory disease in swine and people during a county fair in the United States. Vet Microbiol 137:51-59.

64. Kozak M. 1987. An analysis of 5'-noncoding sequences from 699 vertebrate messenger rNAS. Nucleic Acids Res 15:8125-8148.

65. Sauer B, Handerson N. 1990. Targeted insertion of exogenous DNA into the eukaryotic genome by the Cre recombinase - PubMed. New Biol 2:441-9.

66. Hierholzer JC, Killington RA. 1996. Virus isolation and quantitation, p. 25-46. In Virology Methods Manual. Elsevier.

67. Hain KS, Joshi LR, Okda F, Nelson J, Singrey A, Lawson S, Martins M, Pillatzki A, Kutish GF, Nelson EA, Flores EF, Diel DG. 2016. Immunogenicity of a recombinant parapoxvirus expressing the spike protein of Porcine epidemic diarrhea virus. J Gen Virol 97:2719-2731.

68. Yassine HM, Khatri M, Zhang YJ, Lee CW, Byrum BA, O'Quin J, Smith KA, Saif YM. 2009. Characterization of triple reassortant H1N1 influenza A viruses from swine in Ohio. Vet Microbiol 139:132-139.

69. WHO. 2011. Manual for the laboratory diagnosis and virological surveillance of influenza. WHO Global Influenza Surveillance Network.

70. Dhakal S, Hiremath J, Bondra K, Lakshmanappa YS, Shyu DL, Ouyang K, Kang K il, Binjawadagi B, Goodman J, Tabynov K, Krakowka S, Narasimhan B, Lee CW, Renukaradhya GJ. 2017. Biodegradable nanoparticle delivery of inactivated swine influenza virus vaccine provides heterologous cell-mediated immune response in pigs. J Control Release 247:194-205.

\section{Figure legends}

Figure 1. Construction of ORFV recombinants and their replication kinetics. (A) Schematic representation of homologous recombination between pUC57-121LR-SIV-HA-loxp-GFP plasmid and ORFV-IA82 genome. The recombinant virus was treated with Cre recombinase to remove GFP marker gene to obtain markerless OV-HA construct. (B) Schematic representation of homologous recombination between pUC57-127LR-SIV-NP-loxp-GFP plasmid and OV-HA genome. The recombinant virus was 
739

740

741

742

743

744

745

746

747

748

749

750

751

752

753

754

755

756

757

758

759

760

761

762

763

764

765

766

767

768

treated with Cre recombinase to obtain markerless OV-HA-NP construct. (C) Multi-step (0.1 MOI) and single step (10 MOI) growth curve of OV-HA and OV-HA-NP. OFTu or STU cells were infected with OV-HA and-HA-NP recombinants and virus titers were calculated at $0,6,12,24,48$ and 72 hours post-infection. Error bars represent SEM calculated based on three independent experiments.

Figure 2. Expression of heterologous proteins by ORFV recombinants. (A) Immunofluorescence assay in permeabilized OFTu cells. Upper panel shows expression of HA protein and absence of NP protein in OVHA recombinant. Lower panel shows expression of HA and NP protein by OV-HA-NP recombinant. (B) Immunofluorescence assay performed in non-permeabilized OFTu cells. Upper panel shows expression of HA by OV-HA recombinant and lower panel shows expression of HA and NP by OV-HA-NP recombinant. Blue fluorescence in merged images in panel A and B indicates nuclear staining by DAPI. (C) Expression of heterologous proteins by ORFV recombinants assessed by flow-cytometry. OFTu cells were infected with OV-HA, OV-HA-NP or Wild-type OV-IA82 as negative control. Infected cells were collected 48 hours post-infection, fixed and then stained with appropriate antibodies for flow cytometric analysis.

Figure 3. Immunization-challenge experiment design and humoral response to immunization. (A) A timeline of immunization-challenge experiment. (B) IAV-S specific neutralizing antibody response elicited by immunization with OV-HA and OV-HA-NP. (C) IAV-S specific humoral immune response induced by OV-HA and OV-HA-NP assessed by hemagglutination inhibition (HI) assay. Red arrow heads represent the day of challenge. The error bars represent SEM. VN titer shown in logarithmic scale for effective visualization. $\mathrm{HI}$ titer shown in liner scale. P-values: ${ }^{*} P<0.05,{ }^{*} P<0.01,{ }^{* * *} P<0.001,{ }^{* * * *} P$ $<0.0001$.

Figure 4. IAV-S specific IgG responses to immunization. (A) Total serum IgG level elicited by OV-HA and OV-HA-NP immunization at various time points were assessed by ELISA. Isotype ELISA demonstrating endpoint titers elicited by immunization at 35 days pi in serum was assessed for detecting specific (B) IgG1 and (C) IgG2 antibodies. (D) IgG1/IgG2 ratio in immunized animals was calculated. Each dot represents IgG1/IgG2 ratio of an individual animal. Middle bar represents mean ratio and upper and lower bars represent range. P-values: ${ }^{*} P<0.05,{ }^{*} p<0.01,{ }^{* *} P<0.001,{ }^{* * *} P<0.0001$.

Figure 5. T-cell immune response to immunization. PBMCs isolated from pigs at $35 \mathrm{dpi}$ following recall stimulation with inactivated IAV-S were analysed for: (A) IFN-y production by different T-cell subsets measured by flow cytometry assay; and (B) T-cells proliferation by CFSE dilution assay. Data represents 
769 group means and error bars represent SEM. P-values: ${ }^{*} P<0.05, * * P<0.01,{ }^{* * *} P<0.001, * * * * P<$

$770 \quad 0.0001$.

771 Figure 6. Protective efficacy of OV-HA and OV-HA-NP against IAV-S challenge. (A) IAV-S viral RNA

772 shedding in the nasal swab was determined by RT-qPCR and expressed as log10 genome copy number

773 per milliliter. (B) IAV-S viral load in the lung determined by RT-qPCR and expressed as log10 genome

774 copy number per milliliter. Data represents group mean and error bars represent SEM. P-values: ${ }^{*} P<$

$7750.05,{ }^{* *} p<0.01,{ }^{* * *} p<0.001, * * * * P<0.0001$.

776

777

778

779

780

781

782

783

784

785

786

787

788

789

790

791

792 
bioRxiv preprint doi: https://doi.org/10.1101/2021.04.19.440556; this version posted April 21, 2021. The copyright holder for this preprint (which was not certified by peer review) is the author/funder, who has granted bioRxiv a license to display the preprint in perpetuity. It is made available under aCC-BY-NC-ND 4.0 International license.

\section{Tables}

794 Table 1. Experimental design for immunization-challenge study

\begin{tabular}{|c|c|c|c|c|c|c|}
\hline Group & Immunization & $\begin{array}{c}\text { Number } \\
\text { of animals }\end{array}$ & $\begin{array}{c}\text { Immunization } \\
\text { Days }\end{array}$ & $\begin{array}{c}\text { Immunization } \\
\text { Route }\end{array}$ & Challenge & $\begin{array}{l}\text { Challenge } \\
\text { Dose (Route) }\end{array}$ \\
\hline 1 & Control & 8 & 0,21 & IM & $\begin{array}{c}\text { H1N1 } \\
\text { A/Swine/OH/24366/2007 }\end{array}$ & $\begin{array}{l}6 \mathrm{X} 10^{6} \mathrm{TCID}_{50} \\
\text { (Intranasal) }\end{array}$ \\
\hline 2 & $\mathrm{OV}-\mathrm{HA}$ & 8 & 0,21 & IM & $\begin{array}{c}\text { H1N1 } \\
\text { A/Swine/OH/24366/2007 }\end{array}$ & $\begin{array}{l}6 \times 10^{6} \mathrm{TCID}_{50} \\
\text { (Intranasal) }\end{array}$ \\
\hline 3 & OV-HA-NP & 8 & 0,21 & IM & $\begin{array}{c}\text { H1N1 } \\
\text { A/Swine/OH/24366/2007 }\end{array}$ & $\begin{array}{l}6 \times 10^{6} \mathrm{TCID}_{50} \\
\text { (Intranasal) }\end{array}$ \\
\hline
\end{tabular}

795

796

Table 2. Virus isolation from the nasal swabs

\begin{tabular}{lcccc}
\hline Groups & $\mathbf{0 ~ d p c}$ & $\mathbf{1} \mathbf{d p c}$ & $\mathbf{3 ~ d p c}$ & $\mathbf{7 ~ d p c}$ \\
\hline Control & $0 / 8$ & $4 / 8(50 \%)$ & $7 / 8(87.5 \%)$ & $0 / 8$ \\
OV-HA & $0 / 8$ & $0 / 8$ & $3 / 8(37.5 \%)$ & $0 / 8$ \\
OV-HA-NP & $0 / 8$ & $0 / 8$ & $1 / 8(12.5 \%)$ & $0 / 8$ \\
\hline P-values & - & ${ }^{a} P=0.0769$ & ${ }^{a} P=0.1189$ & - \\
& & ${ }^{b} P=0.0769$ & ${ }^{b} P=0.0101^{*}$ &
\end{tabular}

797

${ }^{\mathrm{a}} \mathrm{P}$-value determined by Fisher's exact test between Control and OV-HA group

${ }^{\mathrm{b}} \mathrm{P}$-value determined by Fisher's exact test between Control and OV-HA-NP group

*Statistically significant difference at $P<0.05$

800

801

802

803

804

805

806

807

808

809

810 
bioRxiv preprint doi: https://doi.org/10.1101/2021.04.19.440556; this version posted April 21, 2021. The copyright holder for this preprint (which was not certified by peer review) is the author/funder, who has granted bioRxiv a license to display the preprint in perpetuity. It is made available under aCC-BY-NC-ND 4.0 International license.

811 Table 3. Pathological and serological findings post-IAV-S-challenge in immunized pigs.

\begin{tabular}{|c|c|c|c|c|c|c|c|c|}
\hline \multicolumn{3}{|c|}{ Control } & \multicolumn{3}{|c|}{ OV-HA } & \multicolumn{3}{|c|}{ OV-HA-NP } \\
\hline $\begin{array}{c}\text { Ani } \\
\text { mal } \\
\text { ID }\end{array}$ & Gross Lesions & VN Titer ${ }^{a}$ & $\underset{\text { ID }}{\text { Animal }}$ & Gross Lesions & VN Titer ${ }^{a}$ & $\underset{\text { ID }}{\text { Animal }}$ & Gross Lesions & $\begin{array}{l}\text { VN } \\
\text { Titer }^{\mathrm{a}}\end{array}$ \\
\hline 22 & $\begin{array}{l}\text { Lobular consolidation } \\
\text { on the left cranioventral } \\
\text { areas }\end{array}$ & $<1: 5$ & 20 & $\begin{array}{l}\text { Mild lobular consolidations on both right } \\
\text { and left lung }\end{array}$ & 1:80 & 21 & No lesions & 1:5120 \\
\hline 23 & $\begin{array}{l}\text { Lobular consolidation } \\
\text { present on ventral areas }\end{array}$ & $<1: 5$ & 24 & No lesions & 1:320 & 25 & No lesions & $1: 2560$ \\
\hline 29 & $\begin{array}{l}\text { Lobular consolidation } \\
\text { mostly present on } \\
\text { cranioventral surface } \\
\text { and interstitial } \\
\text { inflammation on the left } \\
\text { lobe }\end{array}$ & $<1: 5$ & 26 & $\begin{array}{l}\text { Mild lobular consolidation on both sides of } \\
\text { the lung }\end{array}$ & 1:40 & 27 & No lesions & 1:640 \\
\hline 30 & $\begin{array}{l}\text { Lobular consolidation } \\
\text { mostly present on } \\
\text { cranioventral area }\end{array}$ & $<1: 5$ & 33 & No lesions & $1: 640$ & 28 & No lesions & 1:1280 \\
\hline 31 & $\begin{array}{l}\text { Lobular consolidation } \\
\text { mostly present on } \\
\text { cranioventral area }\end{array}$ & $<1: 5$ & 34 & No lesions & 1:2560 & 32 & No lesions & 1:1280 \\
\hline 39 & $\begin{array}{l}\text { Lobular consolidation } \\
\text { mostly present on } \\
\text { cranioventral area }\end{array}$ & $<1: 5$ & 35 & No lesions & 1:1280 & 37 & Very mild lobular consolidation & 1:640 \\
\hline 40 & $\begin{array}{l}\text { Lobular consolidation } \\
\text { mostly present on } \\
\text { cranioventral area }\end{array}$ & $<1: 5$ & 36 & No lesions & $1: 640$ & 38 & $\begin{array}{l}\text { Congestion on apical lobe with mild interstitial } \\
\text { pneumonia }\end{array}$ & $1: 640$ \\
\hline 48 & $\begin{array}{l}\text { Lobular consolidation } \\
\text { on both sides of the } \\
\text { cranioventral area }\end{array}$ & $<1: 5$ & 49 & No lesions & 1:320 & 41 & No lesions & 1:2560 \\
\hline
\end{tabular}

$812 \quad{ }^{a}$ VN titer measured on day 35 post-immunization.

813 All animals were terminated and examined on day 7 post-challenge and evaluated by a pathologist blinded to the study.

814 
bioRxiv preprint doi: https://doi.org/10.1101/2021.04.19.440556; this version posted April 21, 2021. The copyright holder for this preprint (which was not certified by peer review) is the author/funder, who has granted bioRxiv a license to display the preprint in perpetuity. It is made available under aCC-BY-NC-ND 4.0 International license.

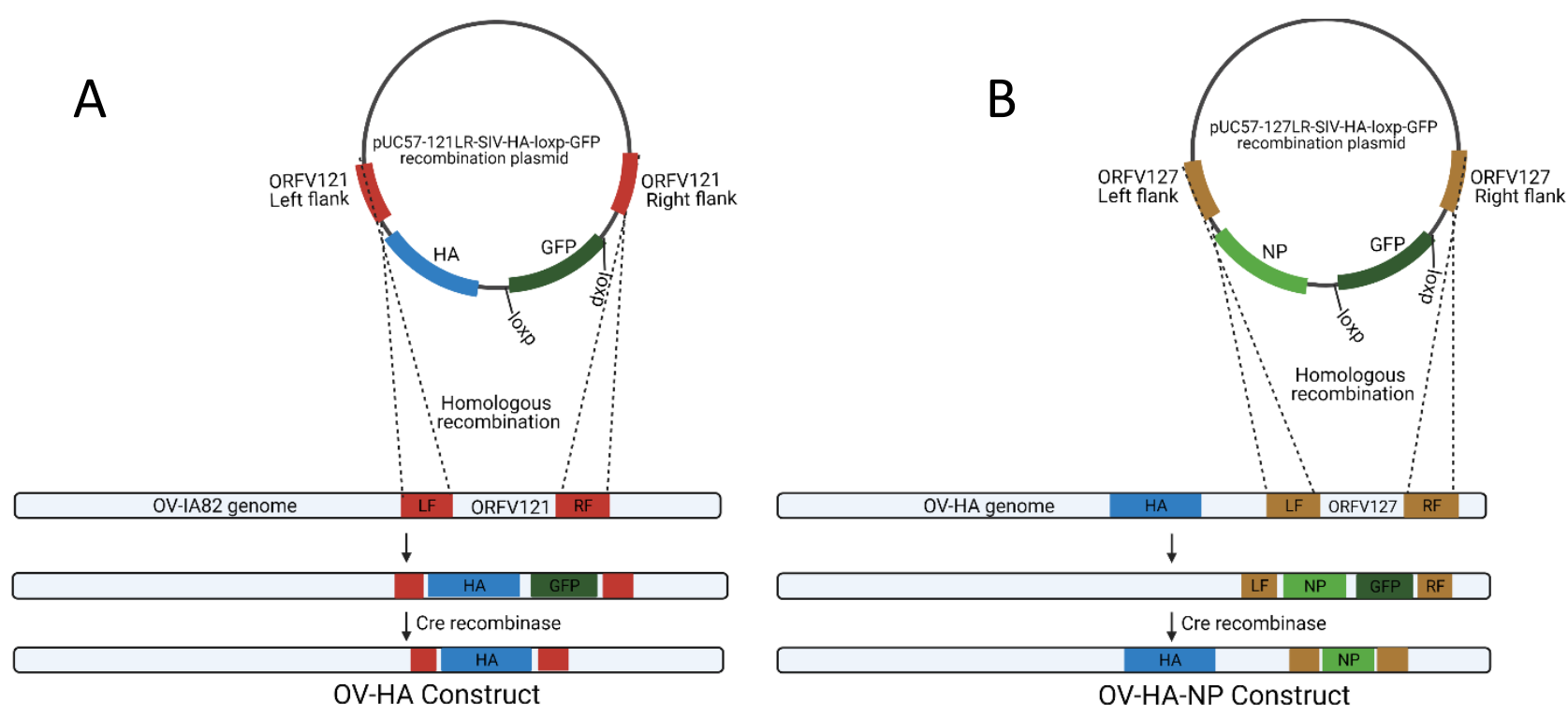

C $0.1 \mathrm{MOI}$

\section{$10 \mathrm{MOI}$}
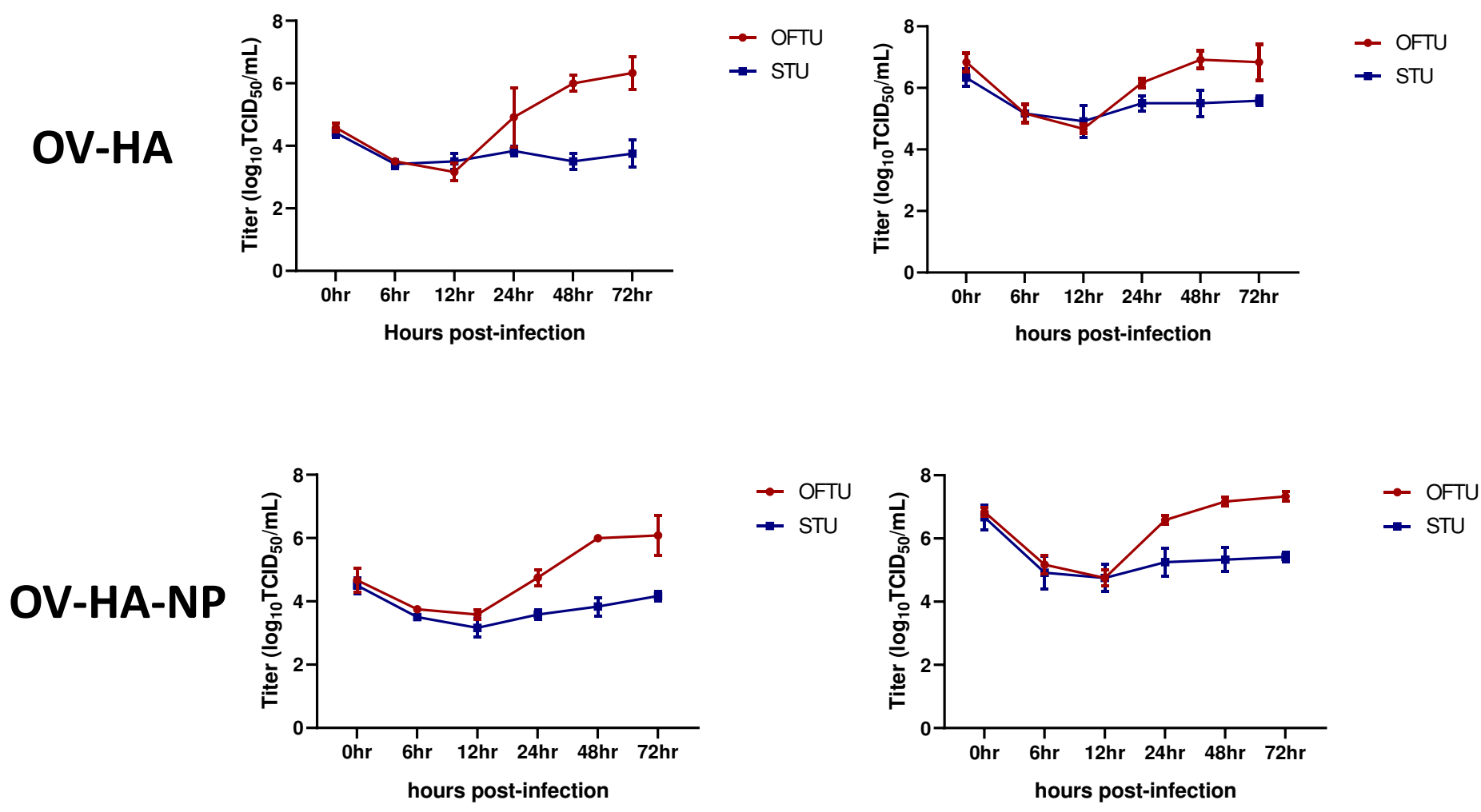

Figure 1 
bioRxiv preprint doi: https://doi.org/10.1101/2021.04.19.440556; this version posted April 21, 2021. The copyright holder for this preprint (which

YAs not certified by peer review) is the author/funder, who has granted bioRxiv a license to display the preprint in perpetuity. It is made

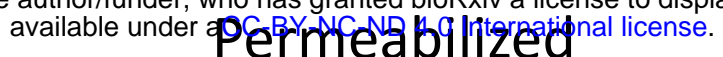
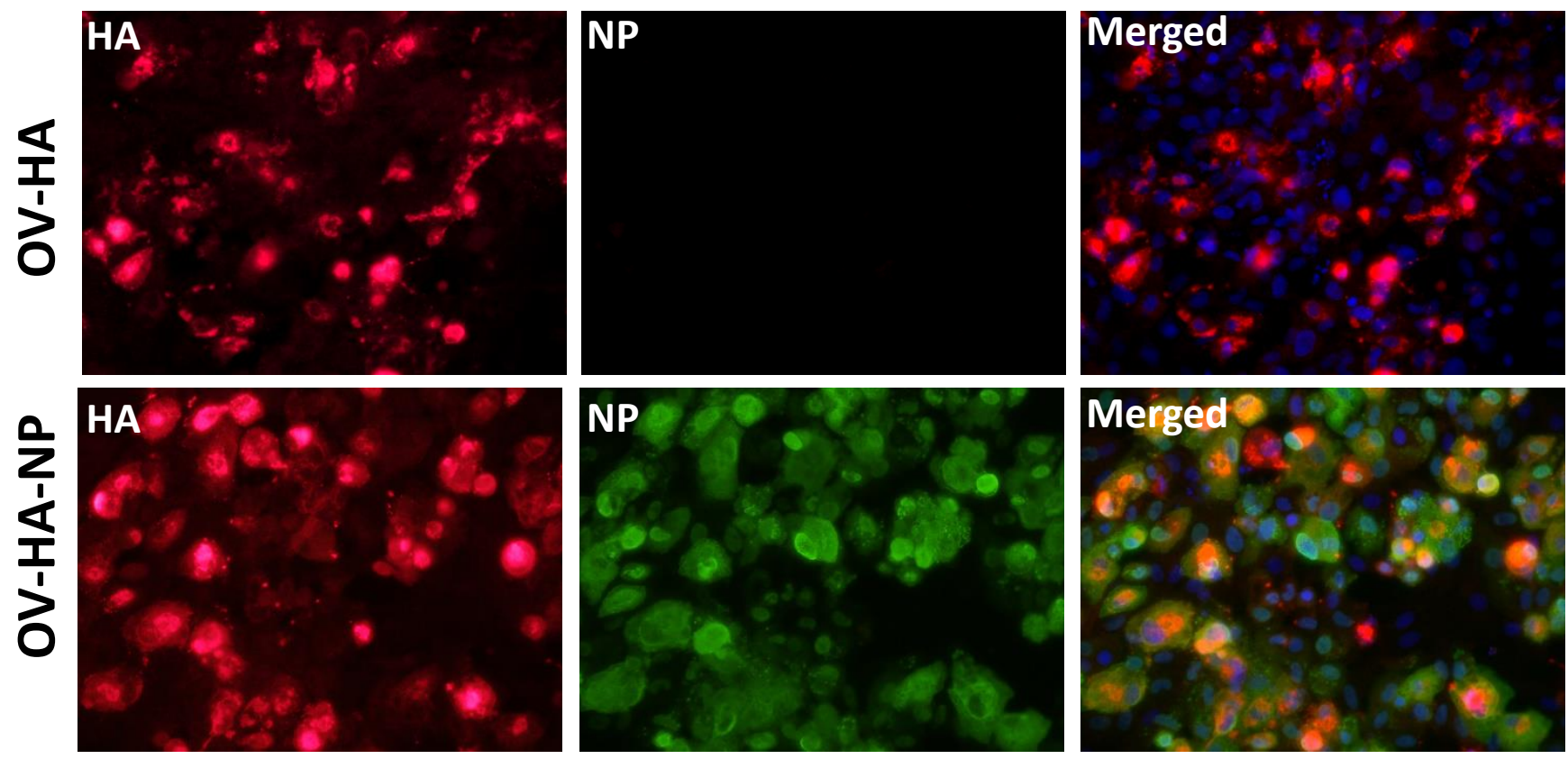

B
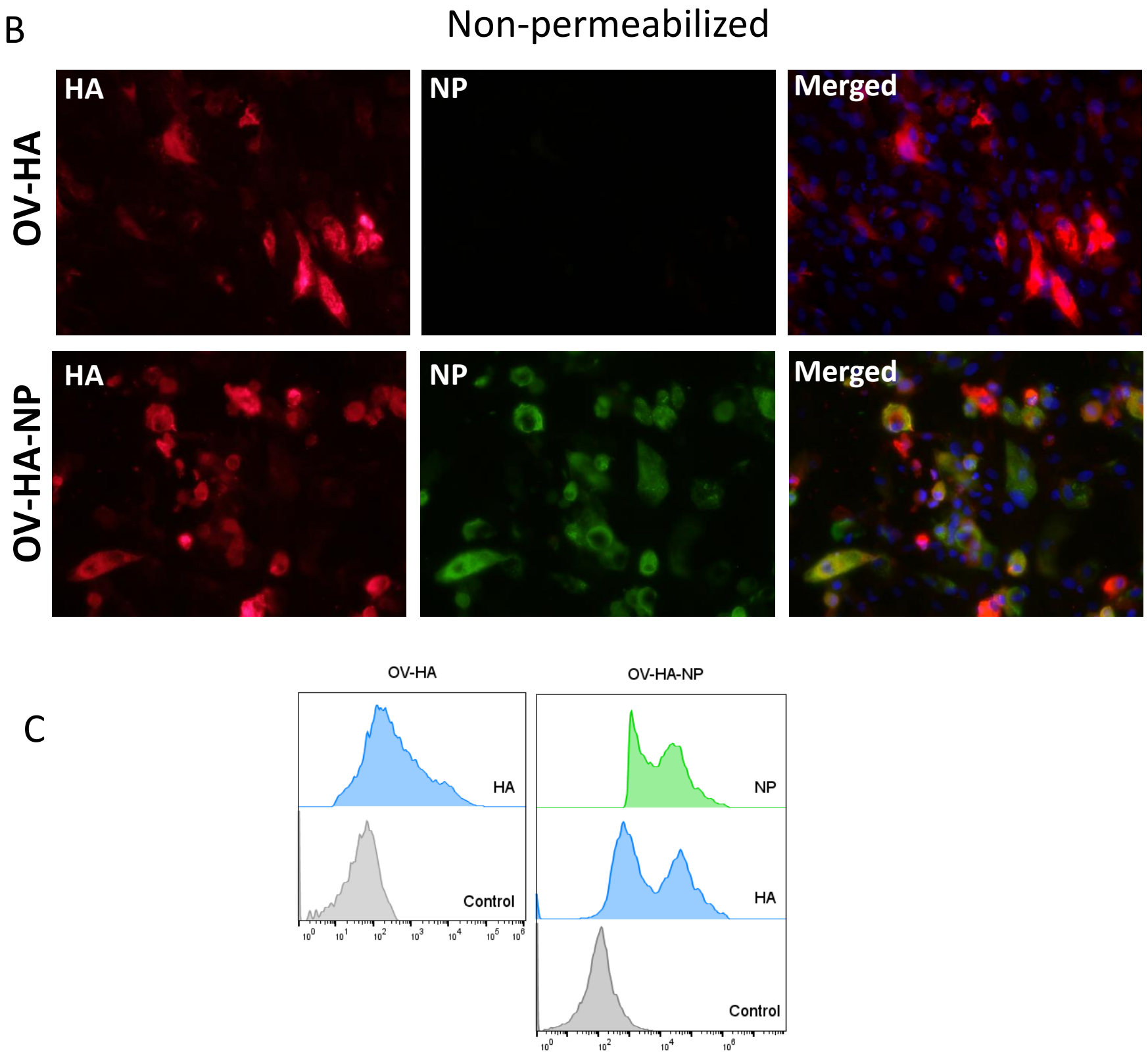

\section{Figure 2}


bioRxiv preprint doi: https://doi.org/10.1101/2021.04.19.440556; this version posted April 21, 2021. The copyright holder for this preprint (which was not certified by peer review) is the author/funder, who has granted bioRxiv a license to display the preprint in perpetuity. It is made available under aCC-BY-NC-ND 4.0 International license.

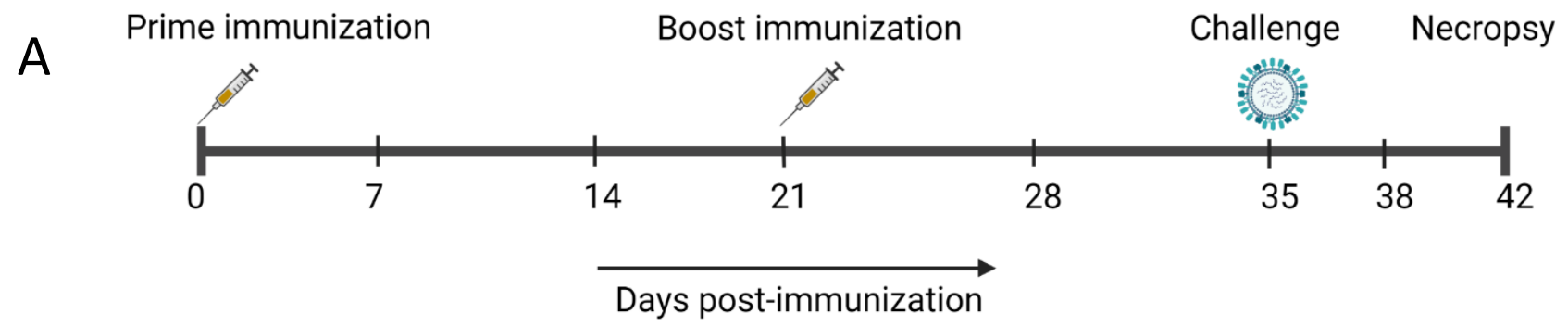

B

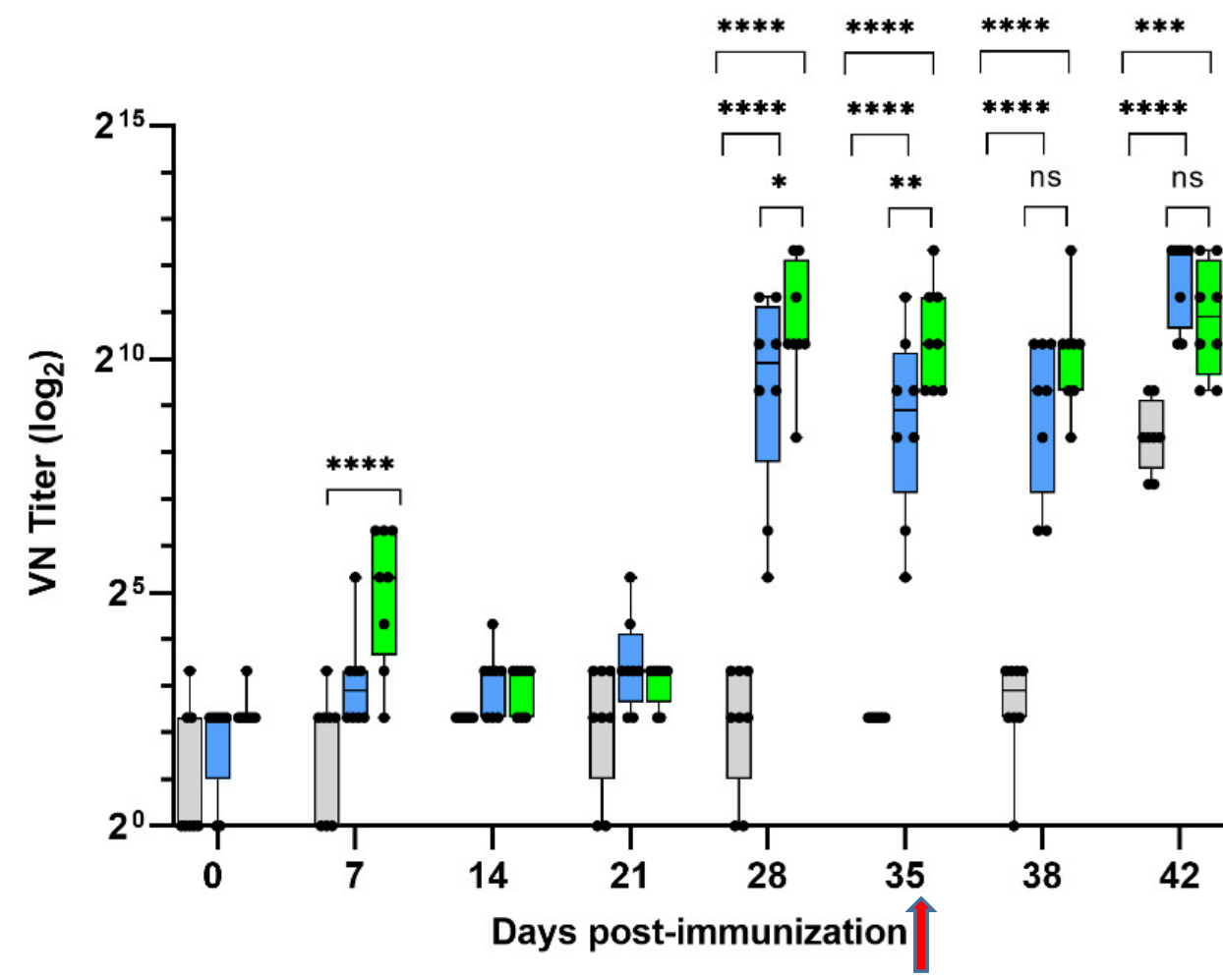

OV-HA

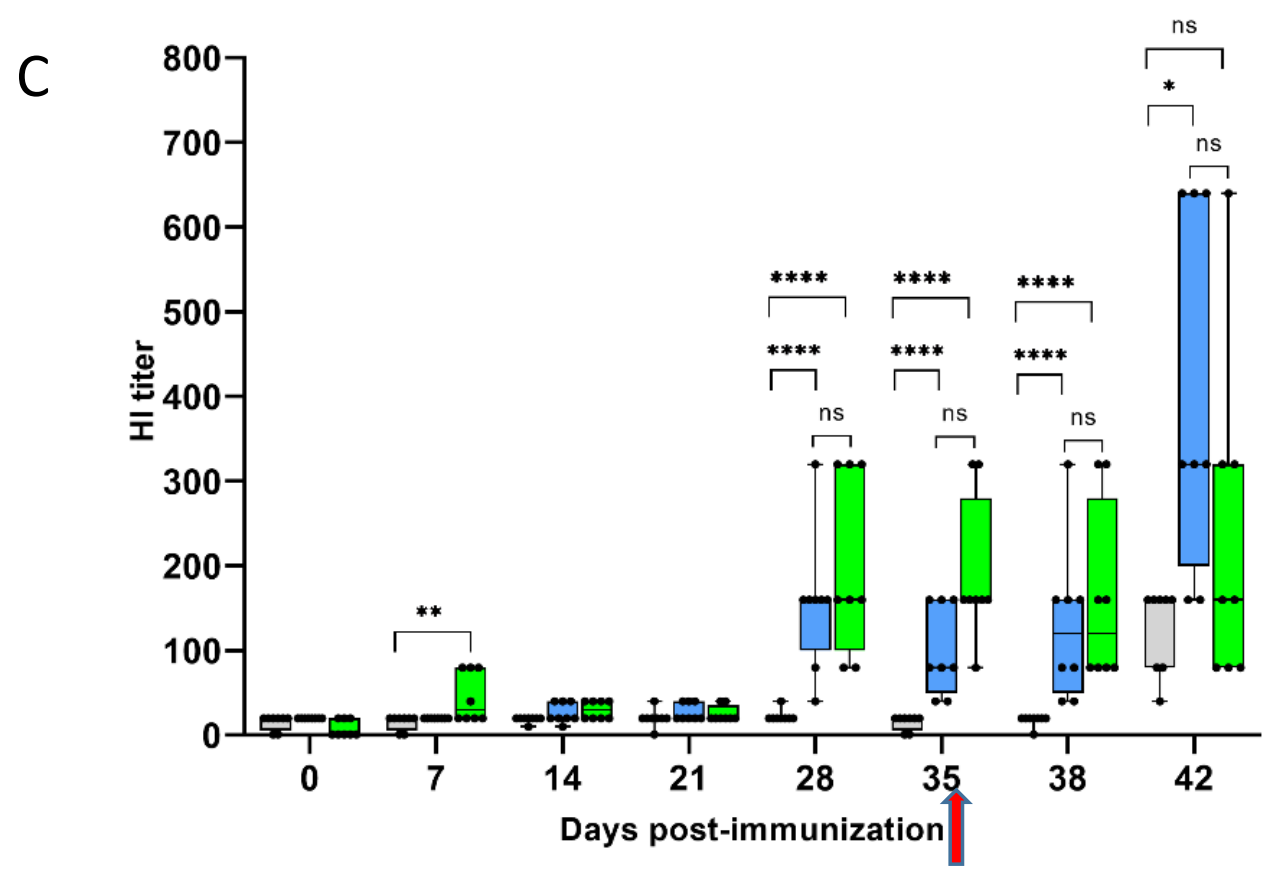

Figure 3 
A
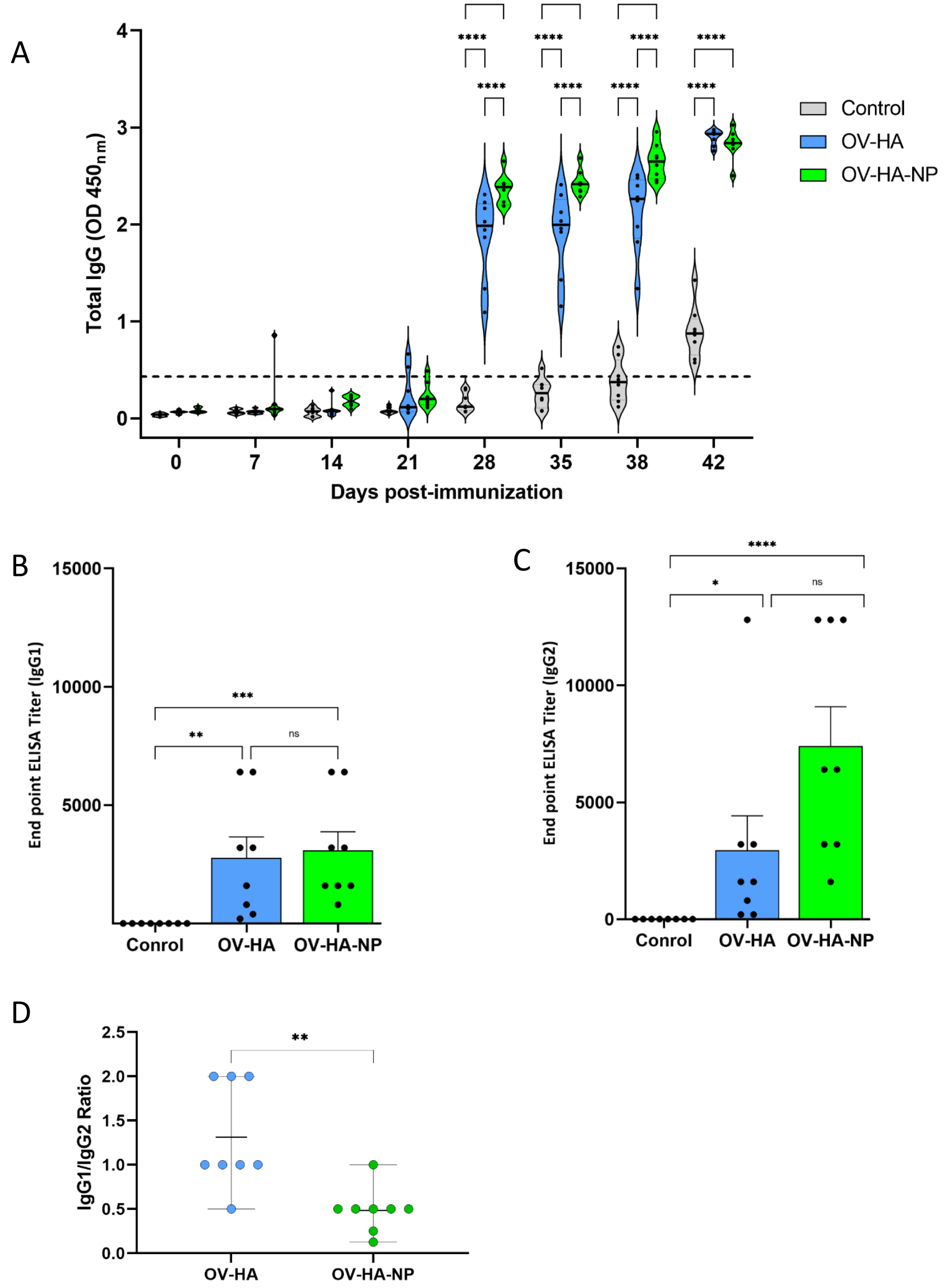

Figure 4 
bioRxiv preprint doi: https://doi.org/10.1101/2021.04.19.440556; this version posted April 21, 2021. The copyright holder for this preprint (which was not certified by peer review) is the author/funder, who has granted bioRxiv a license to display the preprint in perpetuity. It is made available under aCC-BY-NC-ND 4.0 International license.

A
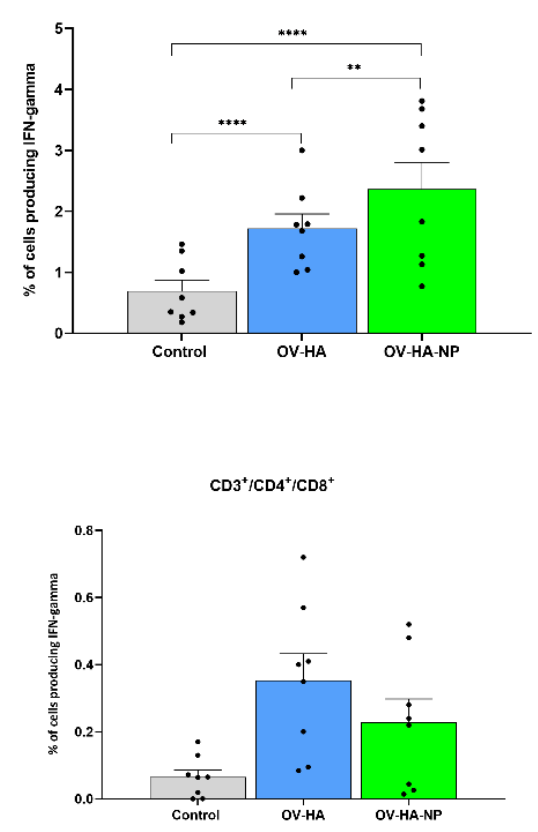

B
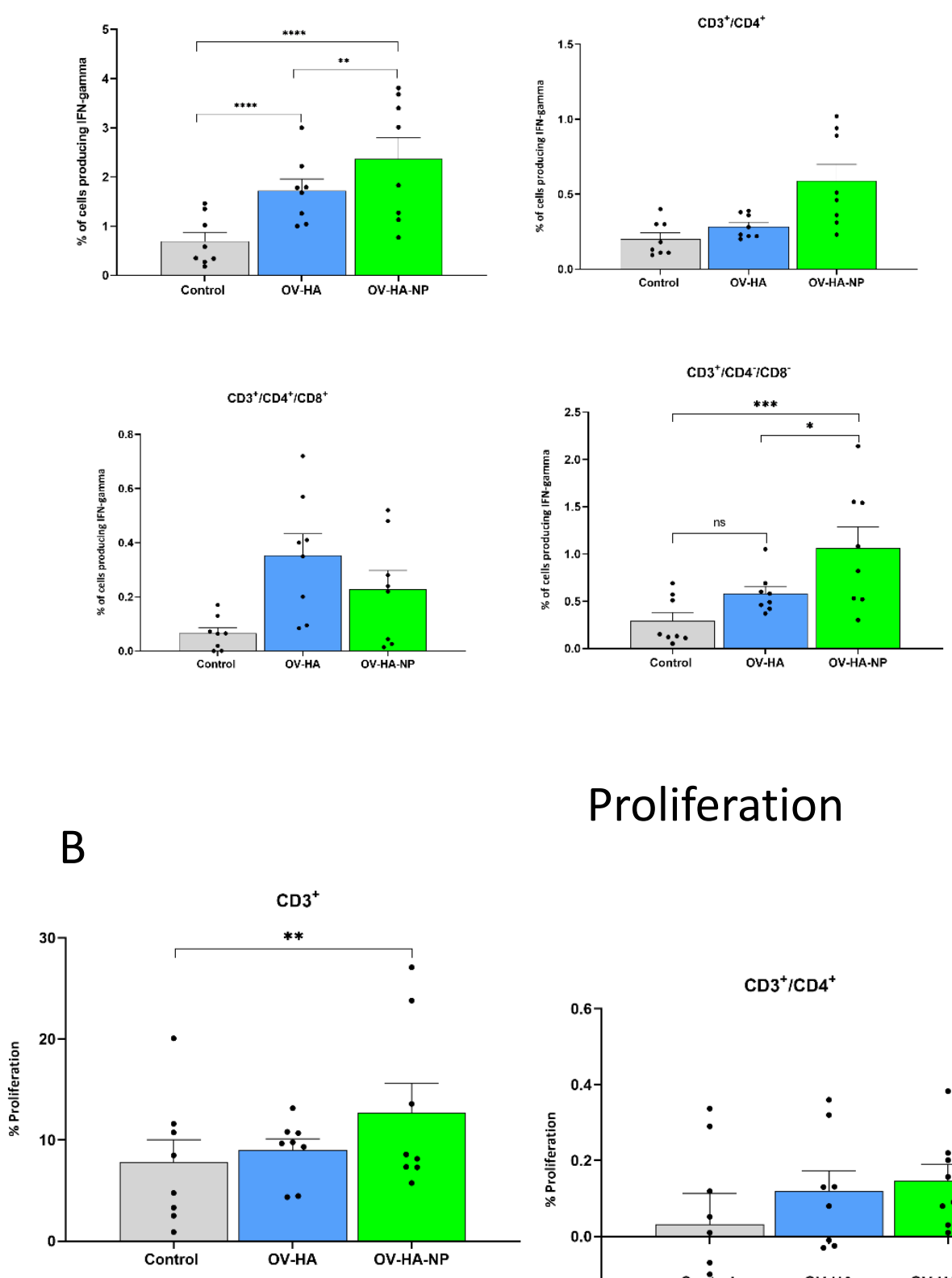

\section{Proliferation}

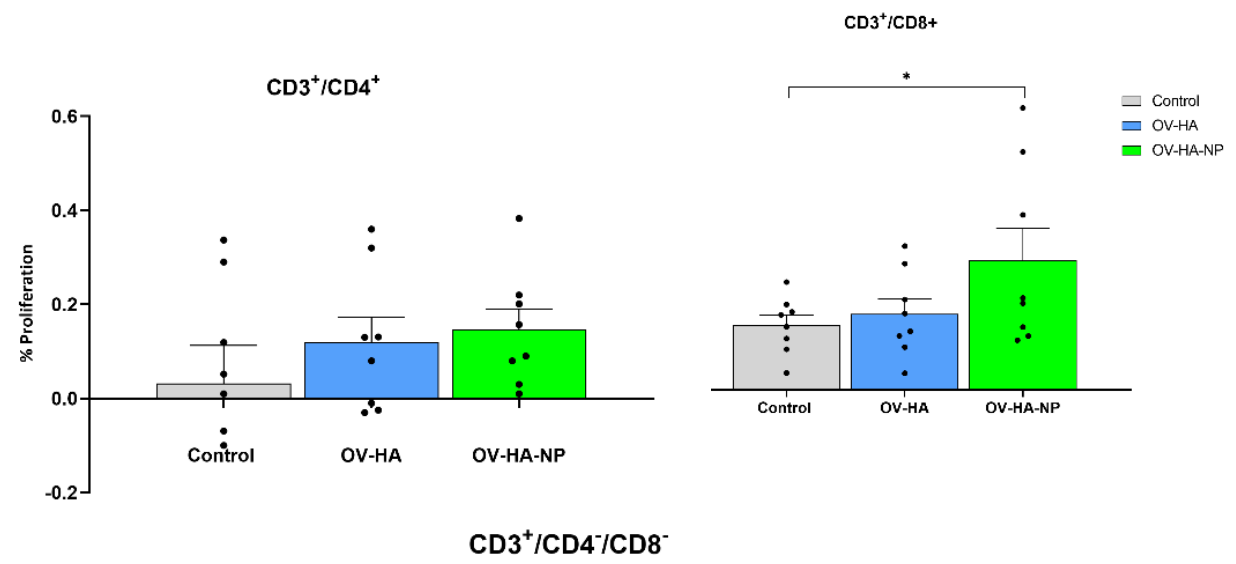

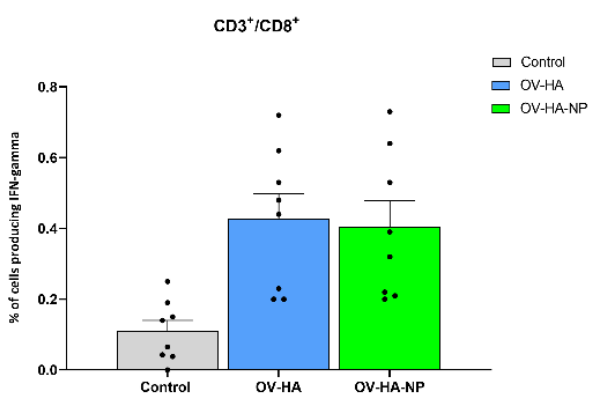

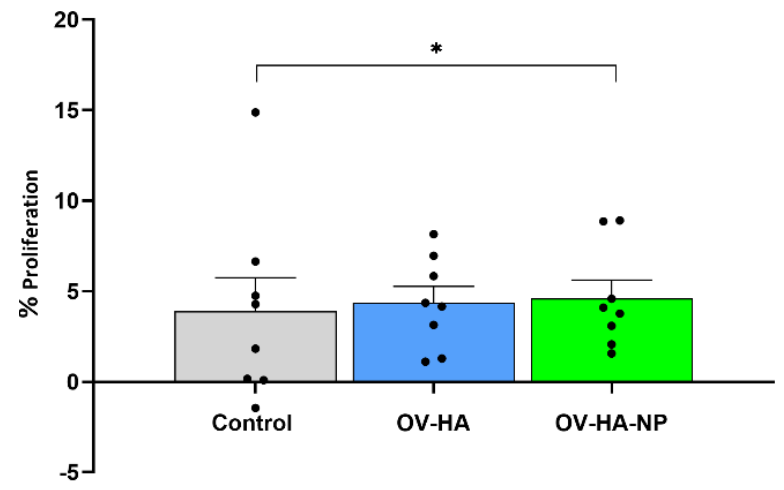


bioRxiv preprint doi: https://doi.org/10.1101/2021.04.19.440556; this version posted April 21, 2021. The copyright holder for this preprint (which was not certified by peer review) is the author/funder, who has granted bioRxiv a license to display the preprint in perpetuity. It is made available under aCC-BY-NC-ND 4.0 International license.

\section{Nasal swab}

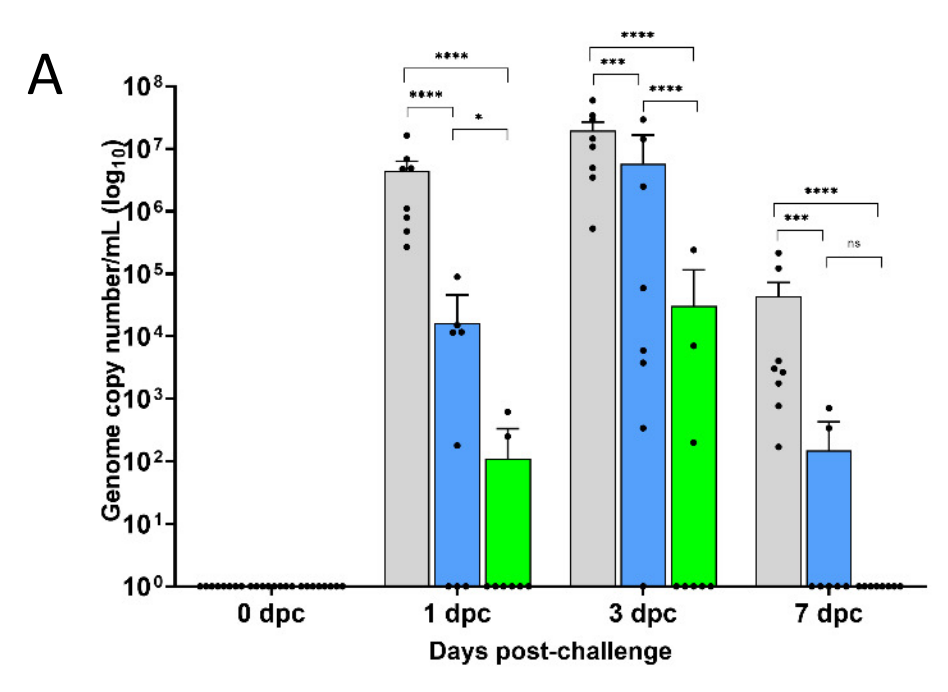

\section{Lung}

B

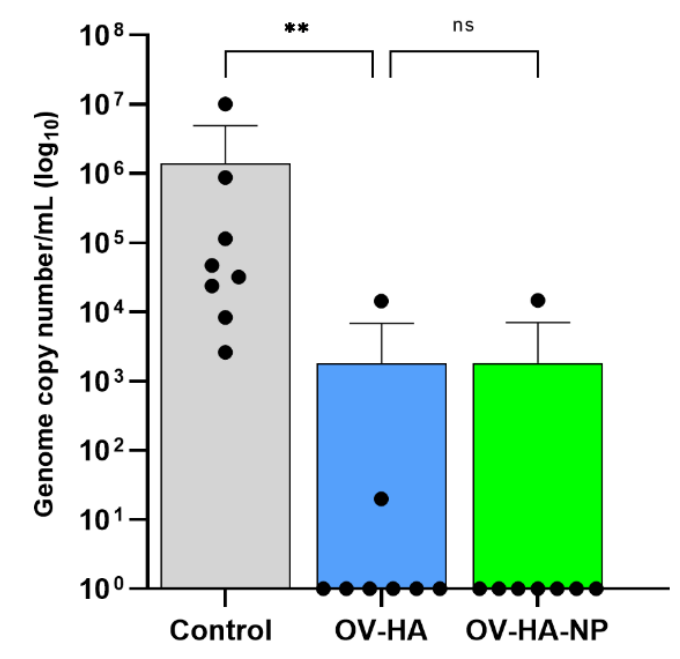

Figure 6 